\title{
Mitochondrial pyruvate carrier abundance mediates pathological cardiac hypertrophy
}

\author{
Mariana Fernandez-Caggiano ${ }^{1 凶}$, Alisa Kamynina1, Asvi A. Francois ${ }^{1}$, Oleksandra Prysyazhna”, \\ Thomas R. Eykyn (102, Susanne Krasemann ${ }^{3}$, Maria G. Crespo-Leiro4,5, Maria Garcia Vieites ${ }^{4}$, \\ Katiuscia Bianchi ${ }^{6}$, Valle Morales ${ }^{6}$, Nieves Domenech ${ }^{4,5}$ and Philip Eaton ${ }^{1 \times}$
}

\begin{abstract}
Cardiomyocytes rely on metabolic substrates, not only to fuel cardiac output, but also for growth and remodelling during stress. Here we show that mitochondrial pyruvate carrier (MPC) abundance mediates pathological cardiac hypertrophy. MPC abundance was reduced in failing hypertrophic human hearts, as well as in the myocardium of mice induced to fail by angiotensin II or through transverse aortic constriction. Constitutive knockout of cardiomyocyte MPC1/2 in mice resulted in cardiac hypertrophy and reduced survival, while tamoxifen-induced cardiomyocyte-specific reduction of MPC1/2 to the attenuated levels observed during pressure overload was sufficient to induce hypertrophy with impaired cardiac function. Failing hearts from cardiomyocyte-restricted knockout mice displayed increased abundance of anabolic metabolites, including amino acids and pentose phosphate pathway intermediates and reducing cofactors. These hearts showed a concomitant decrease in carbon flux into mitochondrial tricarboxylic acid cycle intermediates, as corroborated by complementary $1,2-\left[{ }^{13} C_{2}\right]$ glucose tracer studies. In contrast, inducible cardiomyocyte overexpression of MPC1/2 resulted in increased tricarboxylic acid cycle intermediates, and sustained carrier expression during transverse aortic constriction protected against cardiac hypertrophy and failure. Collectively, our findings demonstrate that loss of the MPC1/2 causally mediates adverse cardiac remodelling.
\end{abstract}

Healthy myocardial mitochondria primarily utilize oxidative phosphorylation to generate adenosine triphosphate (ATP), which is required to meet the heart's energy-demanding function as a blood pump. In healthy myocardium with sufficient oxygen supply, oxidation of fatty acids provides approximately $60-90 \%$ of the myocardial acetyl-coenzyme A that contributes to ATP generation, with $10-40 \%$ arising from pyruvate oxidation ${ }^{1,2}$. However, the stressed human heart changes its fuel preference ${ }^{3,4}$, switching from fatty acids to glucose as a favoured carbon source ${ }^{5,6}$. Consistent with this observation, several studies with animal models of pressure overload-induced hypertrophy have shown reduced fatty acid oxidation rates with enhanced glucose uptake and glycolysis, accompanied by a compensatory anaplerosis to maintain the tricarboxylic acid cycle flux in the pathological heart ${ }^{7-9}$. Interestingly, this enhanced glycolysis and carbon influx via anaplerosis did not augment ATP production, consistent with an 'uncoupling' between glycolysis and glucose oxidation during pathological hypertrophy ${ }^{7,10,11}$. Furthermore, instead of pyruvate predominantly being oxidized in the mitochondria, it is metabolized by alternative pathways, including reductive fermentation to lactate, despite sufficient oxygen availability ${ }^{12,13}$. This is reminiscent of the Warburg effect, whereby many cancer cells increase glucose uptake and convert it to lactate via the reduction of pyruvate despite oxygen availability.

Since the identification of the mitochondrial pyruvate carrier (MPC) in 2012 (refs. ${ }^{14,15}$ ), several studies have reported its role in cell growth and proliferation ${ }^{16-18}$. Mammalian MPC comprises two subunits, MPC1 and MPC2, with the absence of either resulting in the attenuation of mitochondrial pyruvate uptake $\mathrm{e}^{14,15}$. Overexpression of MPC in cancer cells profoundly limited their growth, including in spheroid- or xenograft-implantation models, whereas decreased expression promoted migration, chemotherapy resistance and reduced patient survival ${ }^{19-22}$. Overall this makes a compelling case that decreased MPC abundance may causatively contribute to the Warburg effect and the associated growth potentiation. Consistent with this rationale, we hypothesized that the decreased expression of MPC that we observed in hearts from humans or mice with hypertrophic cardiomyopathy may underlie the switch to anabolism that culminates in maladaptive hypertrophic growth and failure.

Western blot analysis of human hypertrophic cardiomyopathic hearts showed lower MPC1/2 expression than that in control tissue (Fig. 1a). Administration of prohypertrophic angiotensin II to mice for 3 days noticeably reduced cardiac MPC1 and MPC2 expression, which remained low 14 days after treatment initiation (Fig. $1 \mathrm{~b}$ and Extended Data Fig. 1a). MPC expression was similarly attenuated in hearts subjected to cardiac pressure overload involving surgically induced transverse aortic constriction (TAC) for 21 days, which induced hypertrophy (Fig. 1c and Extended Data Fig. 1b).

To investigate whether decreased $\mathrm{MPC} 1 / 2$ expression was causal in the development of cardiac hypertrophy, we developed cardiomyocyte-specific MPC1 or MPC2 transgenic mouse models in which we could increase or decrease the expression of either subunit. However, although the transgenic lines were designed so that MPC1 or MPC2 expression could be individually altered, we found, as have others ${ }^{19,23}$, that increasing or decreasing one subunit resulted in a concomitant matched change in the other subunit.

Knockout (KO) of cardiomyocyte MPC1 or MPC2 separately from birth resulted in complete loss of the other subunit and

\footnotetext{
'The William Harvey Research Institute, Barts and the London School of Medicine and Dentistry, Queen Mary University of London, London, UK. ${ }^{2}$ School of Biomedical Engineering \& Imaging Sciences, King's College London, London, UK. ${ }^{3}$ University Medical Center Hamburg Eppendorf UKE, Institute for Neuropathology, Hamburg, Germany. ${ }^{4}$ Unidad de Cirugia Cardiaca y Trasplante, Servicio de Cardiología, Complejo Hospitalario Universitario de A Coruña (CHUAC), Instituto de Investigación Biomédica de A Coruña (INIBIC), A Coruña, Spain. ${ }^{5}$ Centro de Investigación Biomédica en Red de Enfermedades Cardiovasculares (CIBERCV), Madrid, Spain. ${ }^{6}$ Barts Cancer Institute, Queen Mary, John Vane Science Centre, University of London, London, UK.

凶e-mail: m.f.caggiano@qmul.ac.uk; p.eaton@qmul.ac.uk
} 
a
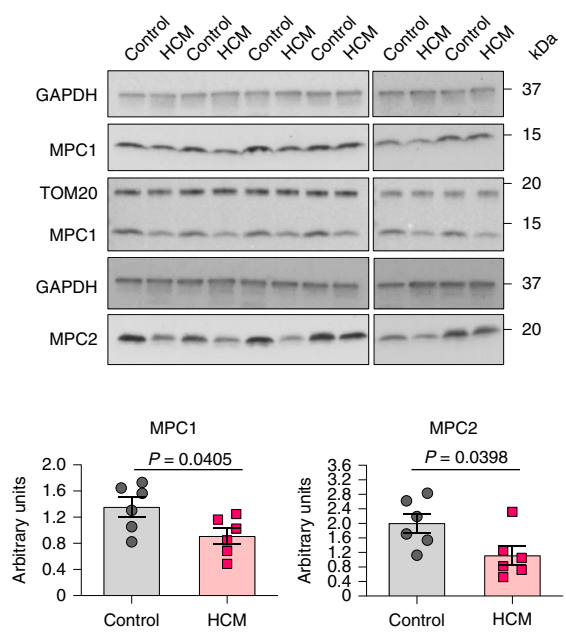

b

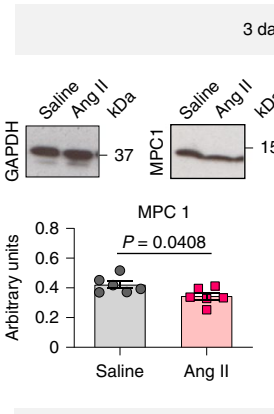

3 days Ang II
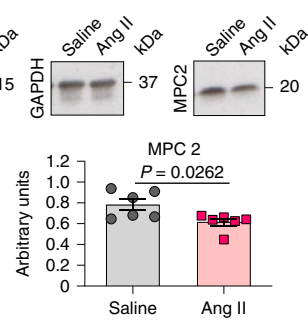

14 days Ang II
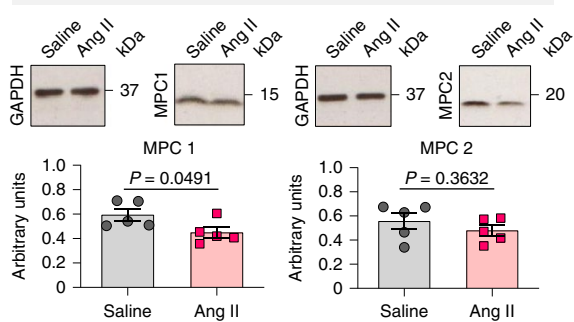

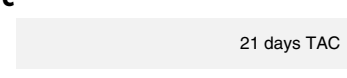

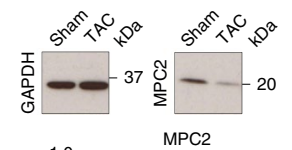
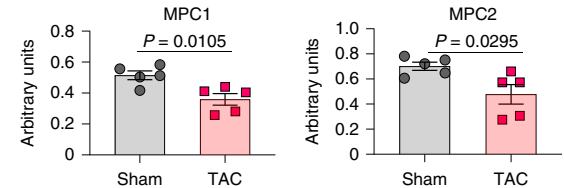

Sham

21 days TAC

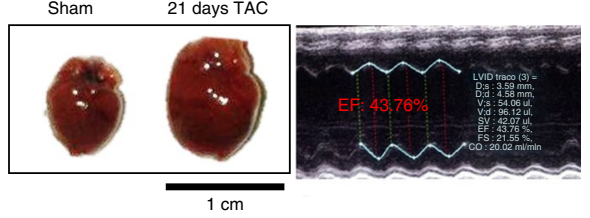

$1 \mathrm{~cm}$

Fig. 1 | MPC1 and MPC2 abundance is lower in hearts of patients with hypertrophic cardiomyopathy and mice induced to undergo pathological hypertrophy. a, Quantitative western immunoblotting analysis shows MPC1 and MPC2 were significantly less abundant in myocardium from patients with hypertrophic cardiomyopathy (HCM) compared with that from donors with a healthy heart (Control), as normalized to GAPDH expression. TOM20 was included as a mitochondrial loading marker control ( $n=6$ biologically independent heart samples per group). b, Quantitative western immunoblotting analysis showed that 3 days of angiotensin II (Ang II) treatment promoted significant loss of cardiac MPC1, as normalized to GAPDH ( $n=6$ biologically independent heart samples per group). Cardiac MPC1 and MPC2 remained lower 14 days after exposure to angiotensin II, as normalized to GAPDH expression ( $n=5$ biologically independent heart samples per group). c, Quantitative western immunoblotting analysis, normalized to GAPDH expression, showed MPC1 and MPC2 were significantly reduced in hearts from mice 21 days after they were subjected to TAC, as compared with sham controls, in which growth was not promoted ( $n=5$ biologically independent heart samples per group). The TAC procedure that decreased MPC1/2 expression also increased hypertrophic growth, as evidenced by a significant increase in heart weight/tibia length ratio, compared with that in the sham group. Statistical significance $(P<0.05)$ was calculated using unpaired, two-tailed, Student's $t$-tests. Data are presented as mean \pm s.e.m.

induced robust heart hypertrophy (Fig. 2a,b). A limitation of studying MPC1 or MPC2 KO mice was their high rate of mortality from 5-6 weeks of age (Fig. 2c). Real-time PCR analysis showed that the cardiac hypertrophy markers, atrial natriuretic peptide (ANP) and brain natriuretic peptide (BNP), were significantly increased in MPC1 $\mathrm{KO}$, as well as in partial MPC1 $\left(\mathrm{MPC1}^{-/+}\right)$mice, whereas $\beta$-myosin heavy chain ( $\beta$-MHC) was significantly increased only in the MPC1 KO mice (Fig. 2d). Partial knockdown of MPC1 from birth to the levels observed in TAC- or angiotensin II-induced hypertrophy also resulted in heart hypertrophy and failure (Fig. 2e and Extended Data Fig. 2).

The observations presented above supported a causal role for decreased MPC abundance in the progression of hypertrophy and heart failure, but the lifelong, constitutive decrease in MPC1/2 expression in the mice studied was a concern. Consequently, we generated a transgenic line (TAXMPC1), in which cardiomyocyte MPC1/2 abundance was inducibly decreased by tamoxifen administration once mice reached adulthood. In these mice, an intraperitoneal administration of tamoxifen promoted the activation of Cre recombinase with the consequent excision of the first exon of the MPC1 gene from the flexible accelerated STOP tetracycline operator (tetO)-knock-in (FAST) cassette only in cardiac myocytes (Fig. 3a). Homozygous (TAXMPC1 KO) or heterozygous $\left(\right.$ TAXMPC $\left.{ }^{+/-}\right)$mice for the FAST cassette showed similar MPC1 and MPC2 protein expression to that in the respective control (Mhy6Cre) in the absence of tamoxifen administration (Fig. 3b). Ten days after tamoxifen injection, the protein expression levels of both subunits were markedly decreased in TAXMPC1 KO and partially decreased in the TAXMPC1 $1^{+-}$line (Fig. 3c). Immunofluorescence analyses of dystrophin expression in heart sections confirmed cardiac myocyte hypertrophy in TAXMPC1 $1^{+-}$mice compared to Mhy6Cre controls 21 days after the initiation of tamoxifen treatment (Extended Data Fig. 4). Cardiac MPC expression in TAXMPC1 ${ }^{+/-}$ mice mimicked the loss observed in angiotensin II- or TAC-treated hearts, and this induced reduction in adult mice alone was sufficient to promote hypertrophy. These hearts had high messenger RNA expression of the hypertrophic markers BNP, ANP and $\beta$-MHC (Fig. 3d), a significant increase in heart weight/tibia length ratio (Fig. 3e), a deterioration in ejection fraction (Fig. 3e) and impaired left ventricular function as illustrated by a reduction in fractional shortening (Fig. 3e). These observations further substantiate that decreased expression of MPC1/2 causatively mediates myocardial growth and progression to heart failure.

Whilst the hypertrophic heart shows enhanced utilization of glucose $^{9}$, its oxidation is unaltered or decreases ${ }^{10,24,25}$. We hypothesized that decreased MPC expression during hypertrophy might causally mediate this 'uncoupling' of glycolysis from glucose oxidation. Indeed, we speculated that loss of MPC directly rewires metabolism to induce hypertrophic remodelling of the stressed myocardium. To test this hypothesis, hearts in which MPC1/2 was inducibly decreased were compared to those with normal, control levels of the carrier using metabolomics profiling by mass spectrometry. This analysis showed that glucose-derived carbon was rerouted, with impaired entry of pyruvate to mitochondria in hearts with less MPC1/2 (Fig. 3f and Supplementary Table 1). Consistent with this, all citric acid cycle intermediates were also less abundant, with decreases in citrate and aconitate reaching statistical significance. Intermediates of glycolysis were also reduced in hearts with 

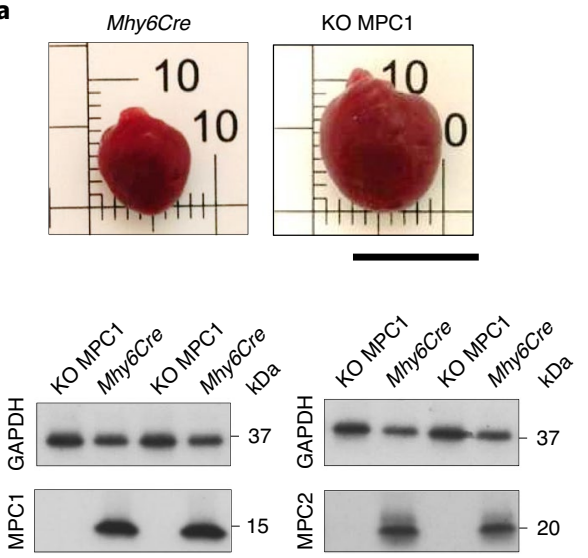

b
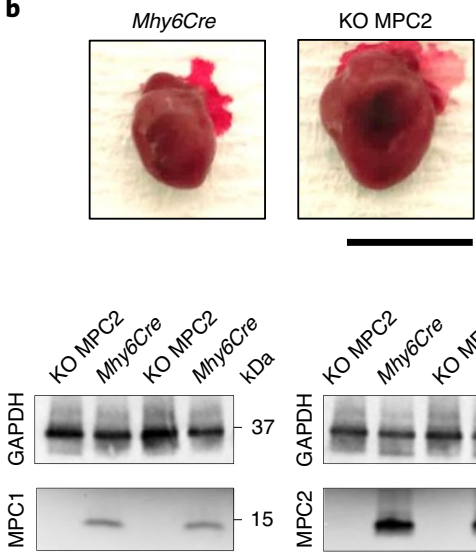

d

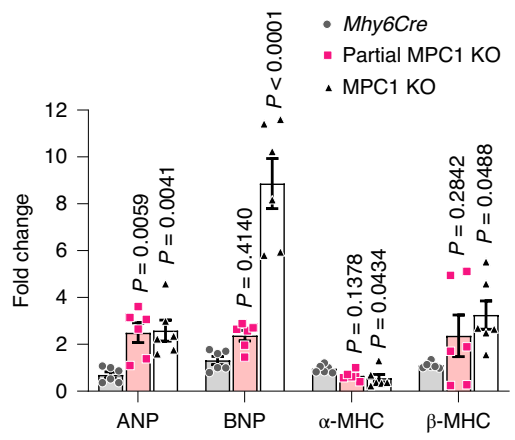
$\quad-\mathrm{BNP}-\mathrm{MHC} \quad \beta-\mathrm{MHC}$

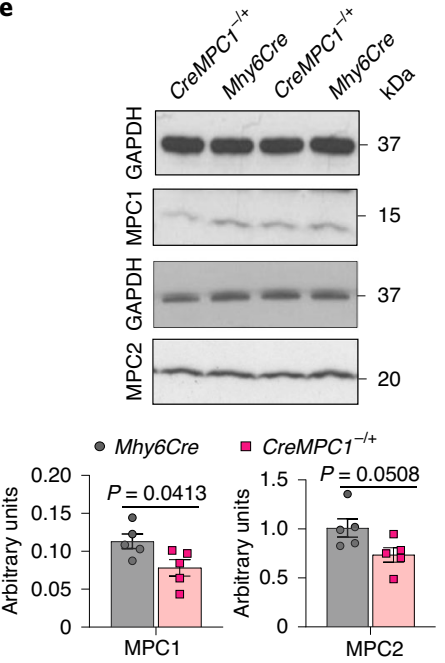

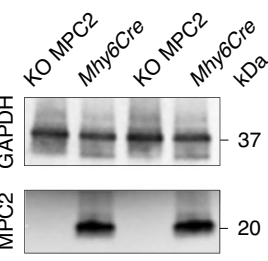

c
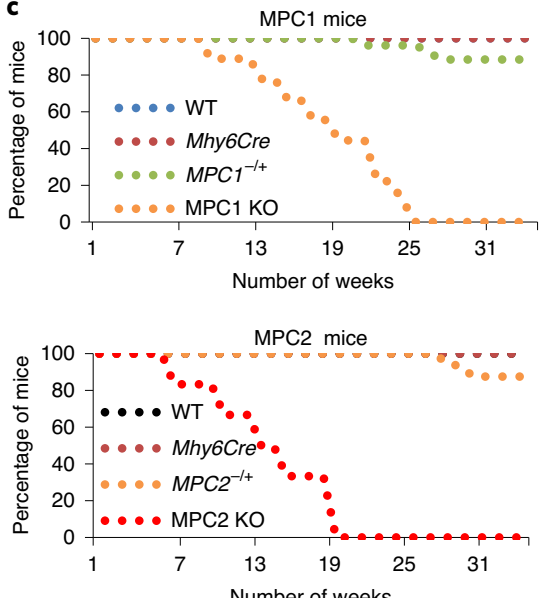
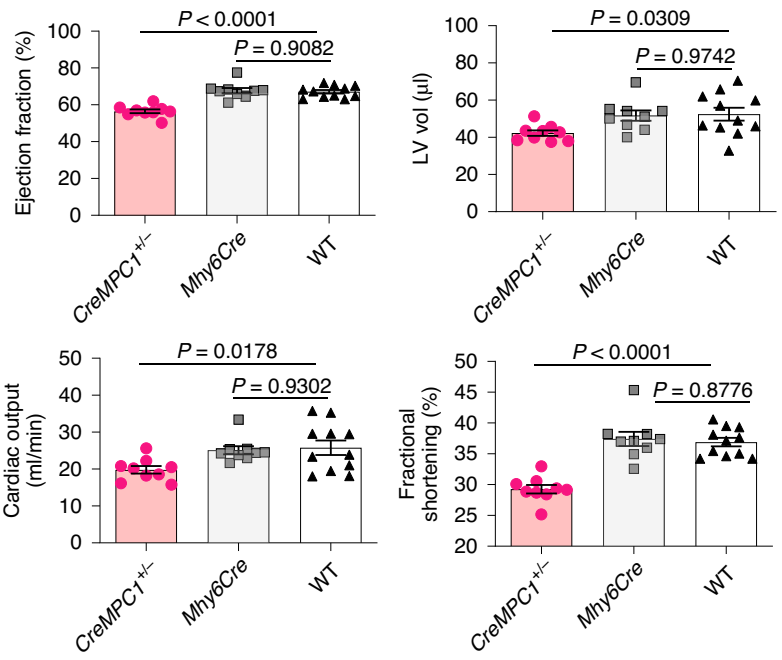

Fig. 2 | MPC1 or MPC2 knockout from birth induces heart hypertrophy and increases mortality. a,b, Quantitative western immunoblotting analysis of MPC1 KO (a) or MPC2 KO (b) hearts showed a reciprocal, concomitant loss of the other subunit of the carrier. This loss of MPC1/2 was associated with a profound increase in cardiac mass. c, MPC1 or MPC2 KO mice in which the MPC gene was deleted from birth showed greater mortality than that in Mhy6Cre control mice, as illustrated by the Kaplan-Meier survival curves, probably because of cardiac failure ( $n=12-20$ mice per group). d, Real-time PCR analysis showed that both the partial MPC1 and MPC1 KO hearts also had significantly increased levels of mRNA hypertrophic markers, namely ANP and BNP, whereas $\beta-M H C$ was only significantly increased in MPC1 KO hearts ( $n=5$ biologically independent heart samples per group). Statistical significance $(P<0.05)$ was calculated using one-way ANOVA adjusted using Dunnett's test for multiple comparisons. e, Partial MPC1 KO $\left(C r e M P C 1^{-/+}\right)$showed lower expression of MPC1 and MPC2 subunits, which was comparable to that in angiotensin II- or TAC-induced hypertrophic hearts. The partial MPC1 KO mice were hypertrophic, and exhibited a reduced ejection fraction, left ventricular volume (LV vol) and fractional shortening $(n=9-10$ mice per group). Statistical significance $(P<0.05)$ was calculated using unpaired, two-tailed, Student's $t$-tests and one-way ANOVA adjusted using Dunnett's test for multiple comparisons. Data are presented as mean \pm s.e.m.

lower MPC1/2 than that in their respective controls, but pyruvate was concomitantly highly increased (Fig. 3f). This increased pyruvate is consistent with impaired entry to the citric acid cycle and not because of enhanced glycolysis. The decrease in glycolytic intermediates was accompanied by rerouting of glucose into the pentose phosphate pathway (PPP), as reflected by significant increases in 6-phosphoglycerate and sedoheptulose 7-phosphate, with ribulose 5-phosphate and ribose 5-phosphate also increasing, albeit not with significance (Fig. 3f). The PPP generates reduced nicotinamide adenine dinucleotide phosphate (NADPH), which is required for fatty acid and membrane biosynthesis, and which was also increased in the MPC KO mice (Fig. 3g). Similarly, 5-carbon ribose 5-phosphate required for nucleotide and nucleic acid production in the hypertrophying heart was increased ${ }^{26,27}$. We performed metabolomic flux tracer analysis of hearts from mice intravenously injected with $1,2-\left[{ }^{13} \mathrm{C}_{2}\right]$ glucose to independently assess, based on the rationale depicted in Extended Data Fig. 5, whether glucose is rerouted to the PPP in myocardium with decreased MPC expression. Indeed, lactate and pyruvates derived from PPP, as evidenced by their single labelling with ${ }^{13} \mathrm{C}$, were significantly increased in $T A X M P C 1^{+/-}$hearts, consistent with rerouting of carbon from glucose into this pathway. Hearts with decreased MPC also concomitantly accumulated more doubly labelled pyruvate and lactate as a result of pyruvate not being incorporated inside the mitochondria and being reduced to lactate (Extended Data Fig. 5). This situation might be explained by a decrease in citric acid cycle metabolite abundance inside the mitochondria (Fig. 3h). Both shunting of glucose-derived carbon into the anabolic PPP and decreased incorporation of pyruvate into the tricarboxylic acid cycle of hearts with decreased MPC provides a rational explanation for the 'uncoupling' of glycolysis from glucose 


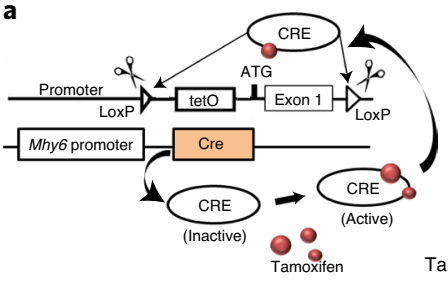

d

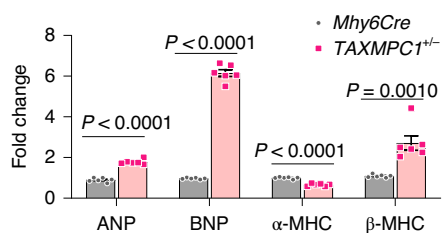

b

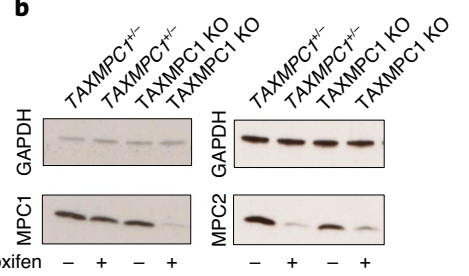

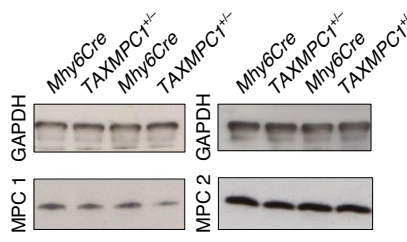
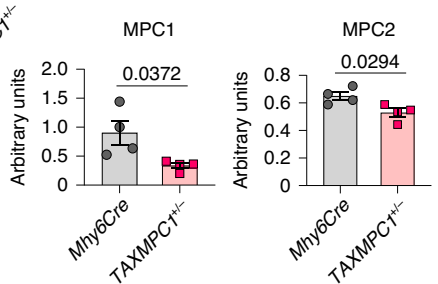

e

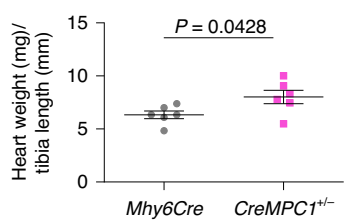

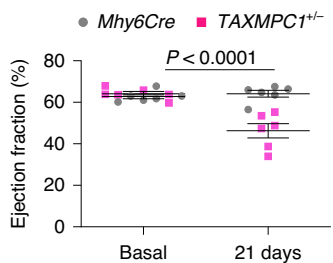

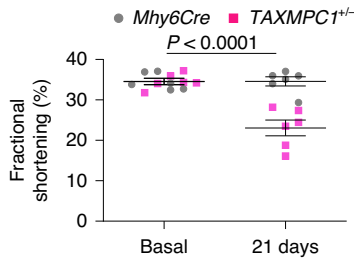

f
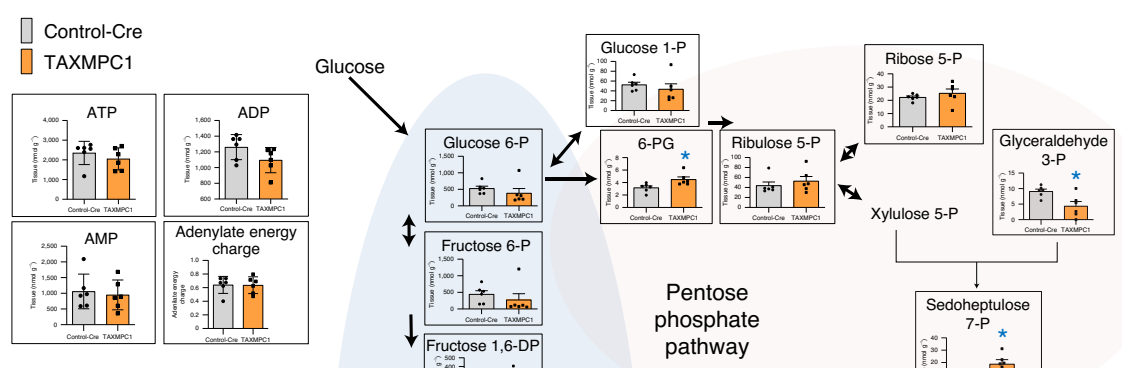

Pentose
phosphate

phosphate
pathway
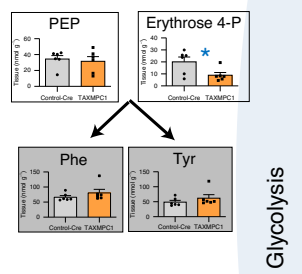

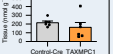
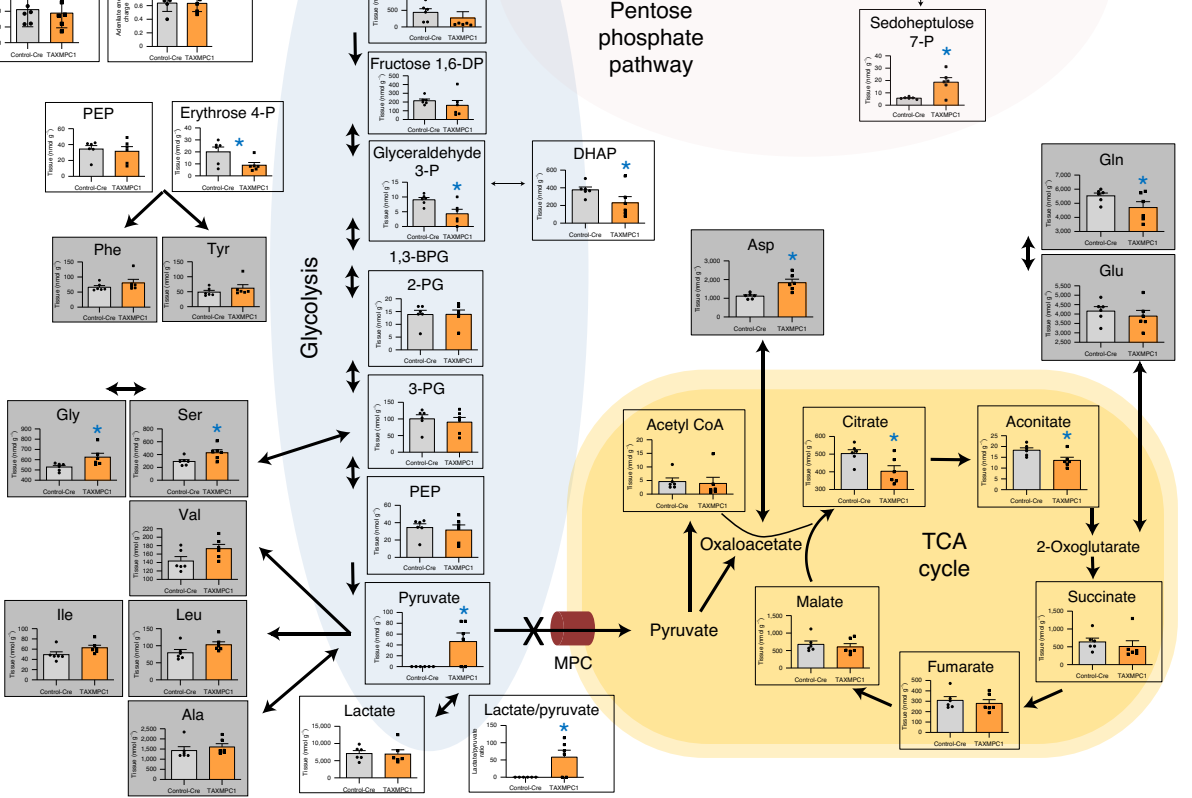

Fig. 3 | Inducibly decreasing MPC expression in adult mice switches metabolism to an anabolic programme that causes hypertrophic growth. a, Design rationale for cardiomyocyte-specific tamoxifen-inducible KO (TAXMPC1) transgenic mice. Intraperitoneal administration of tamoxifen to adult transgenic mice activates Cre recombinase with the consequent excision of the first exon of the MPC1 gene only in cardiomyocytes. $\mathbf{b}$, Western immunoblotting analysis of homozygous (TAXMPC1 KO) or heterozygous ( $T A X M P C 1^{+/-}$) mouse hearts showed that tamoxifen injection to 8-week-old transgenic TAXMPC1 adults promoted a significant reduction in MPC1 and MPC2 protein abundance. c, Western immunoblotting analysis of heart sections from TAXMPC1+/- mice confirmed the anticipated partial reduction in MPC subunit expression 21 days after MPC was inducibly downregulated with tamoxifen ( $n=4$ biologically independent heart samples per group). $\mathbf{d}$, This hypertrophy was corroborated by increases in the mRNAs encoding the hypertrophic markers ANP, BNP and $\beta-\mathrm{MHC}$ in the same samples ( $n=5$ biologically independent heart samples per group). e, Partial MPC knockdown also increased hypertrophy, as indexed by the heart weight/tibia length ratio, resulting in left ventricular dysfunction characterized by decreases in ejection fraction and fractional shortening ( $n=5$ mice per group). f, Pathway analysis obtained from mass spectrometry-based metabolomics of TAXMPC1 KO hearts showed a

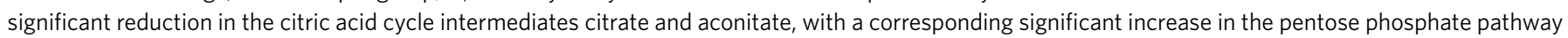
metabolites 6-phosphogluconic acid and 7-sepdoheptulose phosphate ( $n=5$ biologically independent heart samples per group). $\mathbf{g}$, TAXMPC1 KO hearts showed a significant reduction in the ratio NADP/NADPH, whereas there was no difference in the NAD/NADH ratio $(n=5-6$ biologically independent heart samples per group). All statistical significance ( $\left.{ }^{\star} P<0.05\right)$ was calculated using unpaired, two-tailed, Student's $t$-tests. Data are presented as mean \pm s.e.m. 
a

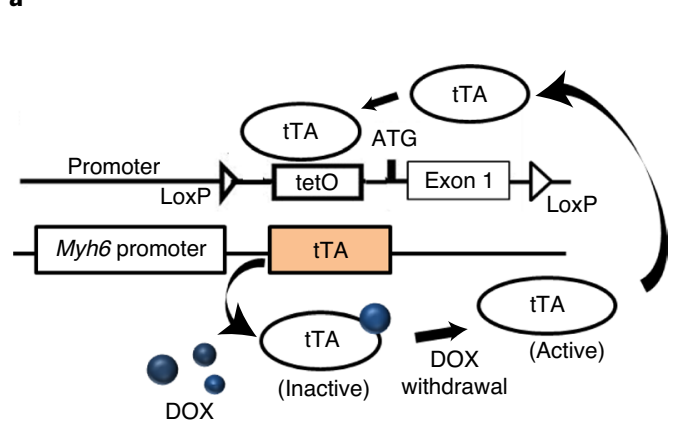

b

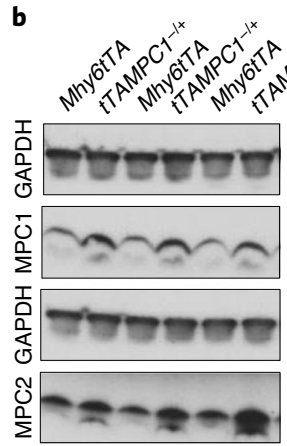

d
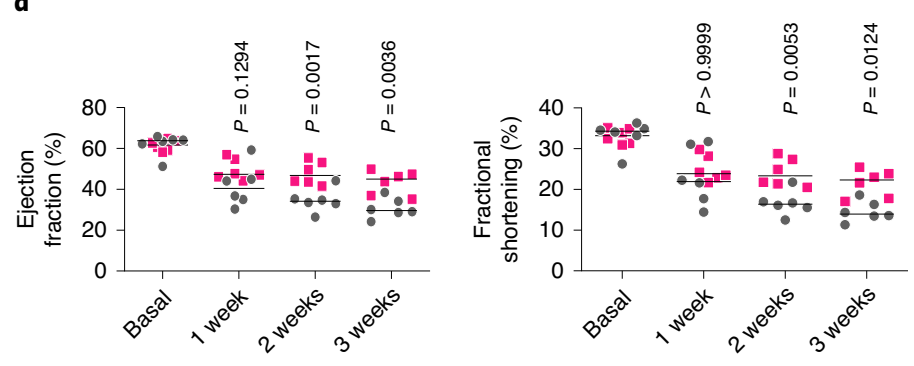
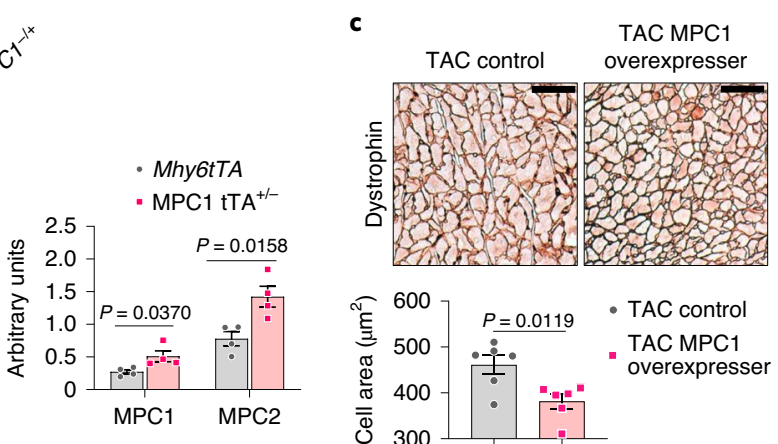

- TAC control

- TAC MPC1 overexpresser

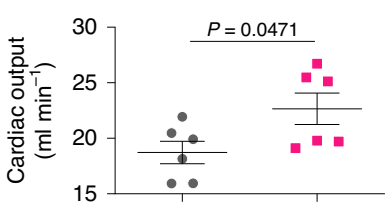

e $\quad$ TAC control

- TAC MPC1 overexpresser

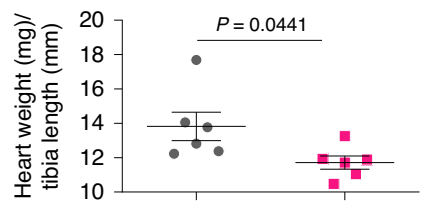

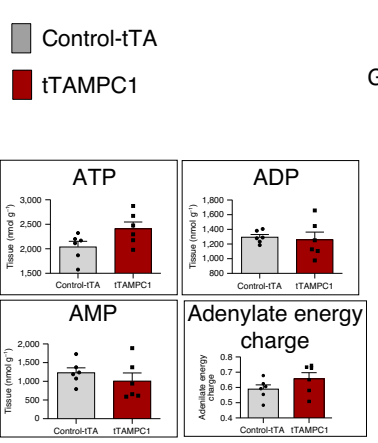
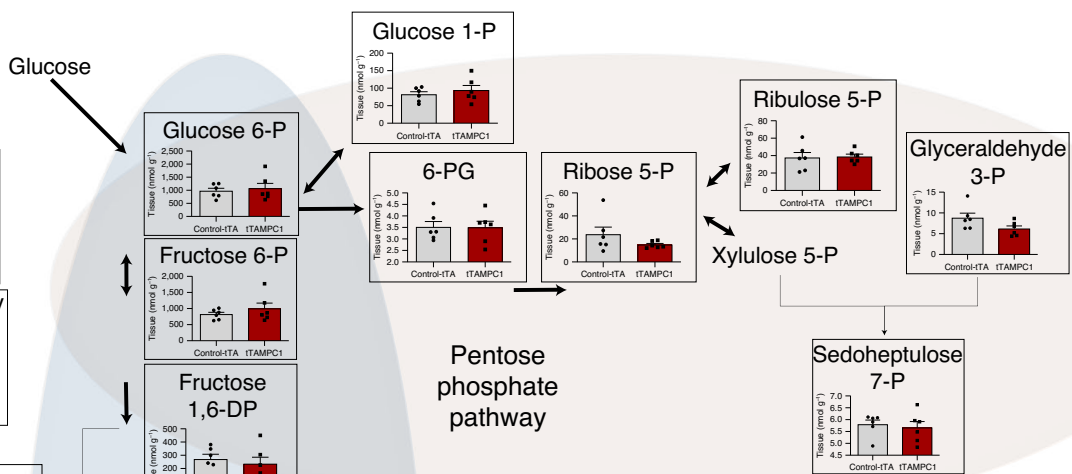

Pentose

phosphate

pathway
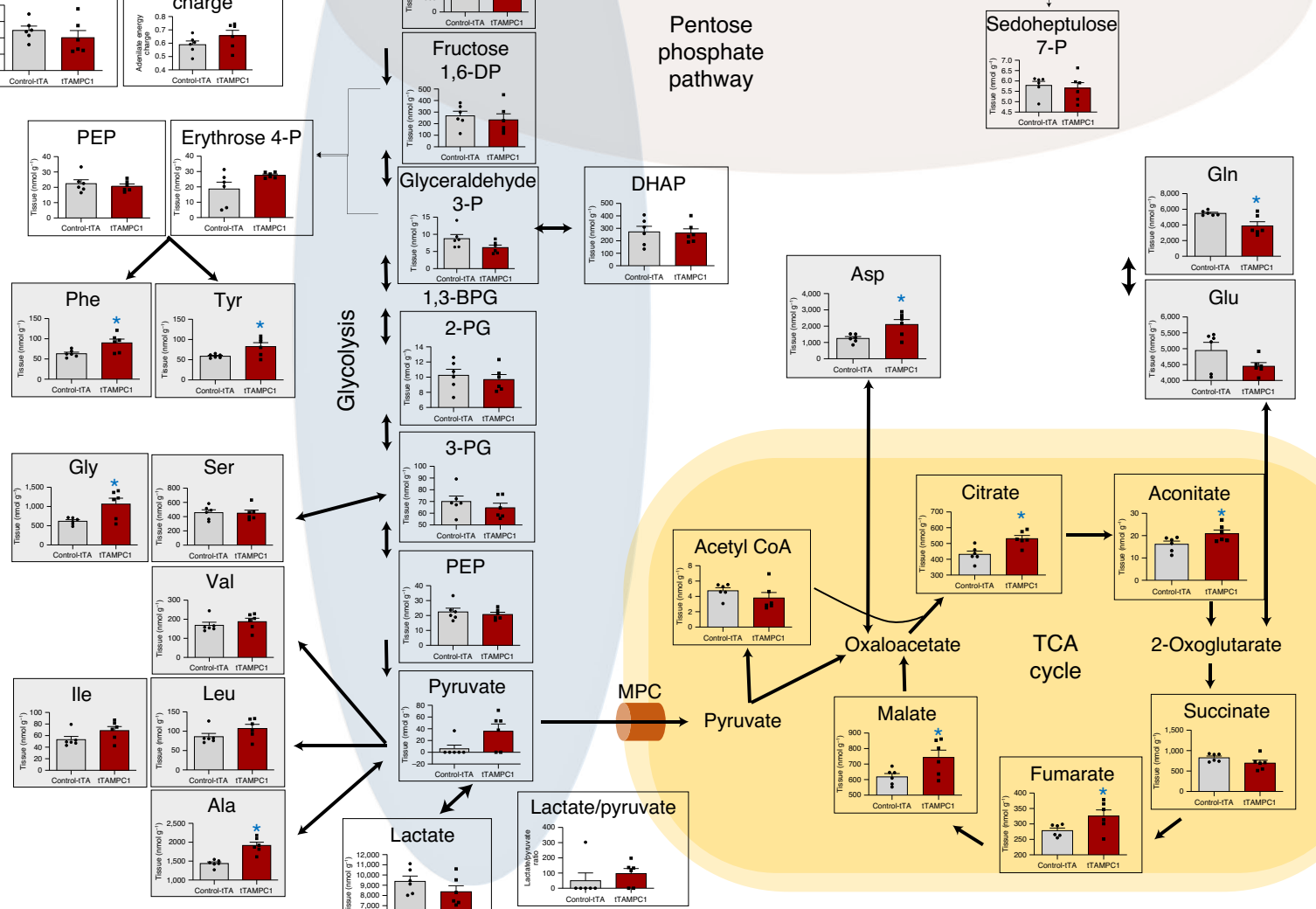

TCA cycle

2-Oxoglutarate

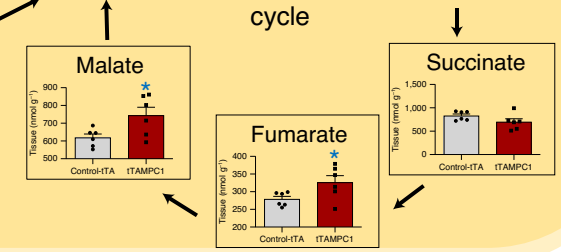


Fig. 4 | Conserved MPC expression in MPC1 overexpressers in a TAC model of cardiac hypertrophy is cardioprotective and limits aberrant growth. a, Overview of the design rationale for inducible cardiomyocyte-specific overexpression of MPC1 (tTAMPC1) or MPC2 (tTAMPC2) transgenic mice. Tetracycline-controlled trans-activator protein (tTA) is inactive in the presence of doxycycline (DOX); however, the withdrawal of this drug induces an efficient interaction between $\mathrm{TTA}$ and the tetO promoter, which should promote enhanced MPC1 or MPC2 transcription. $\mathbf{b}$, Western immunoblotting analysis of hearts from TTAMPC1 mice showed greater MPC1 and MPC2 expression than that in the Mhy6tTA control 21 days after doxycycline withdrawal ( $n=4$ biologically independent heart samples per group). c, Histological measurement of cardiomyocyte surface area in heart tissue sections by immunostaining with dystrophin showed MPC1 overexpression resulted in smaller cardiomyocyte size than that in Mhy6tTA controls in mice subjected to TAC ( $n=150-200$ cells examined over six independent heart samples per group). Scale bar, $100 \mu \mathrm{m}$. d, Echocardiography analysis performed over three consecutive weeks following TAC-induced hypertrophy showed a higher ejection fraction and fractional shortening from week 2 in mice induced to overexpress MPC1 compared to Mhy6tTA controls. Cardiac output was also significantly better than that in controls in MPC1 overexpressers 3 weeks after TAC-induced hypertrophy ( $n=5-6$ mice per group). e. Hypertrophy as indexed by heart weight/tibia length ratio was significantly lower in MPC1 overexpressers compared to controls expressing normal levels of the carrier ( $n=5-6$ mice per group). $\mathbf{f}$, Pathway analysis obtained from mass spectrometry-based metabolomics showed that MPC1 overexpressers significantly increased the citric acid cycle intermediates, including citric acid, aconitate, fumarate and malate ( $n=6$ biologically independent heart samples per group). Statistical significance $\left({ }^{\star} P<0.05\right)$ was calculated using unpaired, two-tailed, Student's $t$-tests for $\mathbf{c}$ and $\mathbf{e}$ and the cardiac output graph in $\mathbf{d}$. Two-way ANOVA adjusted by Bonferroni's multiple comparisons test was use for the other graphs in $\mathbf{d}$. Data are presented as mean \pm s.e.m.

oxidation in the hypertrophic heart ${ }^{10,24,25}$. Furthermore, knockdown hearts had significantly more erythrose 4-phosphate, which couples to aromatic amino acid biosynthesis, explaining the significantly higher phenylalanine and tyrosine in these samples. Additional evidence that reducing pyruvate entry to the mitochondria promotes cardiac growth through enhanced PPP activity came from studies exposing H9C2 cardiomyoblasts to the MPC inhibitor UK5099, which enhanced growth and increased NADPH levels (Extended Data Fig. 6). Although UK5099 has a $K_{\mathrm{i}}$ for the MPC of $<50 \mathrm{nM}$, we used 10 or $20 \mu \mathrm{M}$ based on previous work ${ }^{28}$. Furthermore, we knew from our study with isolated hearts ${ }^{29}$, or from other studies, that exposing cells to micromolar concentrations of UK5099 did not cause toxicity or cell death. ATP and adenylate energy charge in tamoxifen-induced MPC KO hearts 10 days after carrier deletion were the same as in control tissue (Fig. 3f). This is notable, as it demonstrates that the metabolic remodelling that occurs in the hearts of mice with lower MPC1/2 levels occurs independently of ATP abundance (Fig. 3f and Supplementary Table 1). This is consistent with the changes in metabolism upon MPC ablation causatively rerouting glucose carbon into biosynthetic pathways to provide the diverse array of substrates required for myocardial growth. However, additional mechanisms, including those arising from changes in cytosolic redox that occur because of the altered lactate/pyruvate ratio (Fig. 3f), or alterations in pyruvate dehydrogenase activity, may contribute to the remodelling. Ideally we would have performed metabolomics on myocardium from wild-type mice under control as well as TAC conditions, but we did not because of limited resources and because several studies have already reported on this ${ }^{30}$.

If decreased MPC1/2 is critical for cardiac hypertrophy and not simply a bystander effect, maintaining carrier expression should rescue the aberrant growth phenotype. To address this possibility, we generated transgenic mice that allowed MPC1/2 expression to be inducibly maintained at healthy heart levels during stress that would otherwise culminate in loss of carrier expression and hypertrophy.

We generated tTAMPC1 as well as tTAMPC2 cardiomyocytespecific transgenic mouse lines in which doxycycline removal inducibly increases carrier expression. Cardiac-specific tetracycline trans-activator (tTA) transcription factor interacts very efficiently with the tetO promoter in the absence of doxycycline and induces the transcription of MPC (Fig. 4a). MPC1 overexpression was successfully induced upon removal of the drug and showed a parallel increase in MPC2 (Fig. 4b). MPC1 overexpressers subjected to TAC-induced hypertrophy had cardiac myocytes with a significantly smaller cross-sectional cell area (Fig. 4c), as well as improved ejection fraction, cardiac output, fractional shortening and attenuated hypertrophic growth compared with Mhy6tTA controls (Fig. 4d,e). Metabolomics analysis of MPC1 overexpressers showed a robust and significant increase in the citric acid cycle intermediates citrate, aconitate, fumarate and malate, together with a modest increased ATP and adenylate energy charge that would probably improve cardiac energetics and contribute to their resistance to hypertrophy, which is consistent with the anticipated increase in mitochondrial pyruvate uptake (Fig. 4 f and Supplementary Table 2). It is notable that pyruvate, although not statistically significant, was increased in the MPC1 overexpressers. This additional pyruvate could be derived by alanine aminotransferase from alanine, which, for reasons that remain elusive, was significantly increased in the overexpresser. Thus, maintaining MPC expression at the levels found in healthy tissue enhanced mitochondrial pyruvate oxidation and limited the maladaptive myocardial growth and progression to heart failure that occurs during stress induced by pressure overload.

Here, together with two companion articles ${ }^{31,32}$, we conclude that MPC abundance determines the extent to which pyruvate is metabolically oxidized in the mitochondria. Decreasing pyruvate flux into myocardial mitochondria leads to metabolic changes that enable hypertrophy, partly mirroring the Warburg effect, which potentiates cancer cell growth. In contrast, potentiating pyruvate flux into myocardial mitochondria leads to metabolic changes that counteract the hypertrophic growth and progression to heart failure. However, the mechanism underlying MPC downregulation and consequent metabolic remodelling in the failing heart is a key question that remains unanswered. Further investigations of the transcriptional regulation and mechanoresponsive elements that regulate the expression and activity of the carrier are warranted. A better understanding of these pathways may allow interventions that increase MPC abundance or activity to be identified, which are anticipated to decrease pathogenic hypertrophic growth.

\section{Methods}

Study approval. This investigation was performed in accordance with Guidance on the Operation of the Animals (Scientific Procedures) Act 1986 (ref. ${ }^{33}$ ). Animals were maintained humanely in compliance with the 'Principles of Laboratory Animal Care' formulated by the National Society for Medical Research and the Guide for Care and Use of Laboratory Animals ${ }^{34}$. All animal protocols were approved both by the local King's College Ethical Review Process Committee and by the UK Government Home Office (Animals Scientific Procedures Group).

The study was conducted according to Spanish Law for Biomedical Research (Law 14/2007) and was compliant with the Declaration of Helsinki. The study and the use of archive samples for this project were approved by the Research Ethics Committee of Galicia. Written, informed consent was obtained from all individuals involved in this study.

Statistics and reproducibility. Student's $t$-test for unpaired samples was used to compare experiments comprising two experimental groups with normal distribution and equal variance at one time point. One-way analysis of variance (ANOVA) followed by a post hoc Dunnett's test was used to compare cardiac parameters in experiments with more than two groups. Two-way ANOVA followed by a post hoc 
Bonferroni test was used to compare cardiac parameters measured over 21 days in experiments with two groups. Statistical analyses were performed using GraphPad Prism v.5.0 software. Differences were considered significant at $P<0.05$.

Concomitant loss of MPC1 and MPC2, together with a profound increase in cardiac mass, was observed consistently for MPC1 KO or MPC2 KO hearts in more than five independent experiments.

Generation of MPC1 and MPC2 conditional knockout with tetO knock-in mouse models. Transgenic MPC1 and MPC2 FAST mouse lines were generated by Ingenious Targeting Laboratory. Briefly, vectors that contained a LoxP-TetO sequence were inserted immediately upstream of the endogenous ATG initiation site in exon 1 of the MPC1 or MPC2 gene sequence. These vectors were independently electroporated into C57BL/6 embryonic stem (ES) cells. ES clones were selected by resistance to neomycin (Neo), screened and microinjected into blastocysts. Then, blastocysts were implanted into pseudopregnant foster mice. Subsequently, progeny were bred with a flippase recombinase $(\mathrm{C} 57 \mathrm{BL} / 6 \mathrm{NJ})$ transgenic mouse to delete the NEO cassette. Identification of germline transmission of the knockout/knock-in and Neo deletion was performed by PCR

The Ensembl Gene entry for the Mpc1 gene was ENSMUSG00000023861. The FRT-flanked Neo cassette followed by a LoxP site ( $3^{\prime}$ LoxP site) was inserted in intron 1-2 and is about 592 base pairs (bp) away from exon 1 . The Neo insertion site was selected to limit interruption of the promoter region. The construct had a long homology arm that extended about 5.83 kilobases $(\mathrm{kb}) 5^{\prime}$ to the $5^{\prime}$ LoxP site, and the short homology arm is about $2.3 \mathrm{~kb} 3^{\prime}$ to the FRT-Neo-FRT-LoxP cassette.

The Ensembl Gene entry for the Mpc2 gene was ENSMUSG00000026568. The MPC2 gene has multiple transcripts and we focused on transcript Mpc2-001 ENSMUST00000027853. The FRT-flanked Neo cassette followed by a LoxP site ( $3^{\prime}$ LoxP site) was inserted in intron $1-2$, which is about 809 bp away from exon 1. The construct was designed such that the long homology arm extends about $5.3 \mathrm{~kb} 5^{\prime}$ to the $5^{\prime}$ LoxP site, and the short homology arm is about $2.3 \mathrm{~kb} 3^{\prime}$ from the FRT-Neo-FRT-LoxP cassette.

Generation of cardiac-specific MPC1 and MPC2 knockout and overexpressers. Cardiac-specific mouse transgenic lines FVB-Tg(Myh6-cre)2182Mds/J (Jackson Laboratory, stock no. 011037) and FVB.Cg-Tg(Myh6-tTA)6Smbf/J (Jackson Laboratory, stock no. 003170) were back-crossed for $>10$ generations into the C57BL/6NJ male mice background (Jachson Laboratory, stock no. 005304). These mice were then crossed with MPC1 or MPC2 FAST transgenic mice to generate cardiac-specific MPC KO (Mhy6CreMPC1 and Mhy6CreMPC2) or overexpressers (Mhy6tTAMPC1 or Mhy6tTAMPC2). To aid in understanding and simplify the nomenclature relating to the transgenic mice used in these studies, Mhy6CreMPC1 and Mhy6CreMPC2 mice homozygous for the FAST cassette and heterozygous for the Cre cassette were named MPC1 KO and MPC2 KO. Those mice not containing the FAST cassette that were heterozygous for the Cre cassette were used as controls and named Mhy6Cre. In a similar way, Mhy6CreMPC1 and Mhy6CreMPC2 mice homozygous for the FAST cassette and heterozygous for the tTA cassette were named MPC1 overexpresser and MPC2 overexpressers. Mice that did not contain the FAST cassette and were heterozygous for the tTA cassette were used as controls and named Mhy6tTA. MPC overexpressers and their respective tTA controls were fed with a doxycycline diet (Envigo, catalogue no. TD98186) from birth to maintain endogenous expression of the MPC gene. The cardiac-specific $\alpha \mathrm{MHC}-\mathrm{MerCreMer}$ tamoxifen-inducible transgenic line in $\mathrm{C} 57 \mathrm{BL} / 6 \mathrm{NJ}$ male mouse background was provided by M. Zhang (King's College London). These mice were then crossed with MPC1 FAST transgenic mice to generate cardiac-specific tamoxifen-inducible MPC1 KO (Mhy6merCremerMPC1). Mhy6merCremerMPC1 mice homozygous for the FAST cassette and heterozygous for the MerCreMeR cassette were named TAXMPC1. Those mice not containing the FAST cassette and heterozygous for the MerCreMeR cassette were used as controls and named Control-Cre. Intraperitoneal injection $\left(20 \mathrm{mg} \mathrm{kg}^{-1}\right)$ of tamoxifen was performed for five consecutive days to induce deletion of exon 1 of MPC1 and so generate the MPC1 KO. Control-Cre mice were also injected with tamoxifen following the same protocol, which would account for any impact of this drug other than reducing MPC expression.

Unless otherwise stated, male mice between 10 and 13 weeks of age were used for experiments. Animals were kept under pathogen-free conditions, $12 \mathrm{~h}$ lightdark cycle, controlled humidity $(\sim 40 \%)$, temperature $\left(20-22^{\circ} \mathrm{C}\right)$ and fed chow and water ad libitum.

Transverse aortic constriction- and angiotensin II-induced hypertrophy. C57BL/6NJ male mice (body weight $24 \pm 3 \mathrm{~g}, \sim 12$ weeks old) were anaesthetized with isoflurane and placed in the supine position under a dissecting microscope. A 5-mm midline incision was made above the sternal notch to expose the aorta and carotid arteries. The area at both sides of the aorta was cleared and a 7-0 suture was threaded around the aorta between the carotid arteries and tied against a $27 \mathrm{G}$ needle. The needle was then removed, leaving a narrowing of $0.5 \mathrm{~mm}$ in diameter. Animals recovered from anaesthesia in a heated chamber at $28^{\circ} \mathrm{C}$. Control, sham operations were performed by doing the same procedure without tying the knot around the aorta. In some studies, hypertrophy was induced by subcutaneous implantation of osmotic minipumps (Alzet, model 1002) for delivery of angiotensin II (Sigma) at an infusion rate of $1 \mathrm{mg} \mathrm{kg}^{-1} \mathrm{~d}^{-1}$.
Echocardiography was performed with a Vevo 2100 system at heart rates between 400 and 450 beats per minute using a $40-\mathrm{MHz}$ linear probe (VisualSonics v.1.0.0). Mice were anaesthetized with $2 \%$ isoflurane and their body temperature was maintained at $37^{\circ} \mathrm{C}$. Ejection fraction, stroke volume and cardiac output were obtained from high-resolution motion-mode images at the level of the papillary muscle using Vevo Software (VisualSonics v.1.0.0).

Collection of human samples from healthy individuals and patients with hypertrophic cardiomyopathy. Left ventricle biopsies were obtained from the explanted hearts of patients with hypertrophic cardiomyopathy undergoing cardiac transplantation at the A Coruña Hospital. Hypertrophic cardiomyopathy was defined according to the American College of Cardiology and American Heart Association clinical standards. Left ventricle biopsies of healthy hearts were obtained from unused donor hearts from the A Coruña Hospital following the guidelines of Spanish Royal Decrees 2070/1999 and 1301/2006, which regulate the acquisition and use of human tissues for clinical and research purposes. The six individuals with healthy myocardium (four males and two females) had a median age of 52 years. The six patients with hypertrophic cardiomyopathy (three males and three females) had a median age of 52 years.

Immunoblotting analysis. Reduced protein samples prepared from hearts were separated by SDS-PAGE using the Mini-Protean 3 system (Bio-Rad) and transferred to PDVF membranes (Bio-Rad). Blots were incubated with the following primary antibodies diluted 1:1,000 in PBS-Tween 5\% milk overnight: MPC1 (Cell Signaling, catalogue no. 14462), MPC2 (Cell Signaling, catalogue no. 46141) and GAPDH (Cell Signaling, catalogue no. D16H11). One-hour incubation with horseradish peroxidase-coupled anti-rabbit (Cell Signaling, catalogue no. 7074) IgG secondary antibody (diluted 1:1,000 in PBS-Tween 5\% milk) together with enhanced chemiluminescence reagent (GE Healthcare) was used to detect primary antibodies bound to the blot. Blots were cut in line with the $25-\mathrm{kDa}$ band: the bottom half was probed for MPC1 or MPC2, and the upper half was probed for GAPDH as a loading control. The densitometry of western blots was performed using Gel Pro Analyzer v.3.1 (Media Cybernetics).

Real-time PCR for cardiac tissue samples. A 30-mg sample of cardiac tissue was collected, and RNA was extracted using TRIzol reagent (Invitrogen). Using $2-\mu \mathrm{g}$ aliquots of RNA, complementary DNAs were synthesized using the First Strand cDNA synthesis kit (Roche Applied Science) with oligo(dT) primers. DNA primers were designed using Primer3' software (v.0.4.0), and sequences were as follows: $\alpha$-MHC $5^{\prime}$-AGAAGCCCAGCGCTCCCTCA-3' (forward) and 5'-TGCCTCGGGTCAGCTGGGAA-3' (reverse); ANP 5' -TCGTCTTGGC CTTTTGGCT-3' (forward) and 5' -TCCAGGTGGTCTAGCAGGTTCT-3' (reverse); $\beta$-MHC 5'-CCTCGGGTTAGCTGAGAG ATCA-3' (forward) and 5'-ATGTGCCGGACCTTGGAAG-3' (reverse); BNP 5'-AAG TCCTAGCCA GTCTCCAGA-3' (forward) and 5'-GAGCTGTCTCTGGGCCATTTC-3'(reverse); GAPDH 5' -CATGGCCTTCCGTGTTCCTA-3' (forward) and 5' -CCTGCTTCA CCACCTTCTTGAT-3' (reverse).

All primers were loaded at $600 \mathrm{nM}$, and $1 \mu \mathrm{l}$ of cDNA was amplified by duplicate in $10 \mu \mathrm{l}$ of $1 \times$ SYBR Green Mix (Roche Applied Science) using a ABI7900 HT thermocycler (ThermoFisher Scientific). The PCR was set up as follows: a denaturing step of $7 \mathrm{~min}$ at $94^{\circ} \mathrm{C}$, followed by 35 amplification cycles of $94^{\circ} \mathrm{C}$ for $10 \mathrm{~s}, 60^{\circ} \mathrm{C}$ for $10 \mathrm{~s}$ and $72^{\circ} \mathrm{C}$ for $10 \mathrm{~s}$. PCR products were tested for melting temperature with a temperature ramp of $2.2^{\circ} \mathrm{C} \mathrm{s}^{-1}$ from 65 to $95^{\circ} \mathrm{C}$ and shown to correspond to the expected cloned fragments. GAPDH was used as a reference, and relative expression was estimated by the $\Delta \Delta \mathrm{Ct}$ method as the average value of each sample normalized against the average value of control hearts.

\section{Steady-state metabolomics using capillary electrophoresis-mass spectrometry} analysis. Approximately $50 \mathrm{mg}$ of frozen tissue was plunged into $1.5 \mathrm{ml}$ of $50 \%$ acetonitrile/Milli-Q water containing internal standards (Human Metabolome Technologies, catalogue no. $\mathrm{H} 3304-1002)$ at $0{ }^{\circ} \mathrm{C}$ to inactivate enzymes. The tissue was homogenized three times at 1,500 r.p.m. for $120 \mathrm{~s}$ using a tissue homogenizer (Microsmash MS100R, Tomy Digital Biology) and then the homogenate was centrifuged at $2,300 \mathrm{~g}$ and $4{ }^{\circ} \mathrm{C}$ for $5 \mathrm{~min}$. Subsequently, $800 \mu \mathrm{l}$ of upper aqueous layer was centrifugally filtered through a Millipore 5 -kDa cut-off filter at $9,100 \mathrm{~g}$ and $4{ }^{\circ} \mathrm{C}$ for $120 \mathrm{~min}$ to remove proteins. The filtrate was centrifugally concentrated and re-suspended in $50 \mu \mathrm{l}$ of Milli-Q water for capillary electrophoresis-mass spectrometry (CE-MS) analysis.

Metabolome measurements were carried out by Human Metabolome Technologies (HMT). Peaks detected by CE-TOFMS and CE-MS/MS were extracted using automatic integration software (MasterHands, Keio University, and MassHunter Quantitative Analysis B.04.00, Agilent Technologies, respectively) to obtain peak information, including $m / z$, migration time (MT) and peak area. The peaks were annotated with putative metabolites from the HMT metabolite database based on their MTs in CE and $m / z$ values determined by TOFMS. The tolerance range for the peak annotation was configured at $\pm 0.5 \mathrm{~min}$ for MT and $\pm 10 \mathrm{ppm}$ for $\mathrm{m} / \mathrm{z}$. In addition, concentrations of metabolites were calculated by normalizing the peak area of each metabolite with respect to the area of the internal standard and by using standard curves, which were obtained by three-point calibrations. 
${ }^{13} \mathrm{C}$ flux metabolomics using LC-MS analysis. Approximately $50 \mathrm{mg}$ of frozen heart was ground in a mortar submerged in liquid nitrogen. Metabolites were extracted using a solution with $50 \%$ methanol, $20 \%$ acetonitrile and $30 \% \mathrm{H}_{2} \mathrm{O}$. Liquid chromatography-mass spectrometry (LC-MS) analysis of the extracted samples was performed using a Q Exactive Hybrid Quadrupole-Orbitrap mass spectrometer coupled to a Vanquish UHPLC system (ThermoFisher Scientific). The liquid chromatography system was fitted with a Sequant ZIC-pHILIC column $(150 \mathrm{~mm} \times 2.1 \mathrm{~mm})$ and guard column $(20 \mathrm{~mm} \times 2.1 \mathrm{~mm})$ from Merck Millipore and the temperature was maintained at $35^{\circ} \mathrm{C}$. The mobile phase was composed of $10 \mathrm{mM}$ ammonium carbonate and $0.1 \%$ ammonium hydroxide in water (solvent $\mathrm{A}$ ) and acetonitrile (solvent B). The flow rate was set at $100 \mu \mathrm{min}^{-1}$ with the gradient described previously ${ }^{35}$. The mass spectrometer was operated in full MS and polarity switching mode. The acquired spectra were analysed using XCalibur Qual Browser and XCalibur Quan Browser software v.4.2 (ThermoFisher Scientific)

Dystrophin immunohistochemistry. Heart tissues were fixed in $4 \%$ formalin-PBS and embedded in paraffin using an ASP300S dehydration machine (Leica) and an EG1160 tissue embedding system (Leica). Immunohistochemical staining was performed as follows. Sections were cut at a thickness of $2 \mu \mathrm{m}$. After dewaxing and inactivation of endogenous peroxidases (PBS-3\% hydrogen peroxide), antibody-specific heat-mediated antigen retrieval was performed using the Ventana Benchmark XT machine. Sections were blocked (PBS-10\% FCS) and afterwards incubated with anti-dystrophin antibody (1:200; EMD Millipore, catalogue no. 1645). For detection of specific binding, the Ultra View Universal DAB Detection Kit (Ventana, Roche) was used, which contains secondary antibodies, DAB stain and counter-staining reagent for detection of nuclei. Representative images were taken with a Leica DMD108 digital microscope. Cardiomyocyte area quantification was analysed using ImageJ (v.1.51w).

NADP/NADPH and NAD/NADH assays. NADP ${ }^{+} / \mathrm{NADPH}$ and NAD/NADH ratios were measured using the $\mathrm{NADP}^{+} / \mathrm{NADPH}-\mathrm{Glo}$ and $\mathrm{NAD}^{+} / \mathrm{NADH}-\mathrm{Glo}$ assay kits (Promega), respectively, following the manufacturer's instructions. Briefly, $5 \times 10^{6} \mathrm{H} 9 \mathrm{C} 2$ cells or $30 \mathrm{mg}$ of heart tissue were lysed in $200 \mu \mathrm{l}$ or $300 \mu \mathrm{l}$, respectively, of base solution (Sigma). Half of the lysate was treated with $0.4 \mathrm{~N} \mathrm{HCl}$ at $60^{\circ} \mathrm{C}$ to remove the $\mathrm{NADH}$ and $\mathrm{NADPH}$ to allow measurement of $\mathrm{NAD}^{+}$and $\mathrm{NADP}^{+}$. Cell and tissue lysates were mixed with kit detection reagents and after $30 \mathrm{~min}, \mathrm{NADP}^{+}$/ NADPH ratios were measured using a CLARIOstar Plus (BMG Labtech).

Real-time cell proliferation analysis. The xCELLigence system (RTCA SP, Acea Biosciences) was used for real-time and time-dependent analysis of H9C2 cell responses to UK5099 $(10 \mu \mathrm{M}$ or $20 \mu \mathrm{M})$. A total of 20,000 cells were seeded on a PET 16 E-Plate and cultured in $250 \mu$ of DMEM Glutamax (ThermoFisher Scientific). After $24 \mathrm{~h}$, cells were treated with vehicle or UK5099 and the real-time cell index was continuously measured for the following $96 \mathrm{~h}$ using the cell impedance detection system. Cell index is a measure of impedance that correlates with the number, attachment and size of the cells.

Reporting Summary. Further information on research design is available in the Nature Research Reporting Summary linked to this article.

\section{Data availability}

The data that support the findings of this study are available from the corresponding author on request. Human Metabolome Technologies uses the Human Metabolome Database (HMDB) (https://hmdb.ca/). The metabolomic data and the HMDB link for each metabolite are available online as Supplementary Table 1 and Supplementary Table 2. Source data for Figs. 1-4 and Extended Data Figs. 1-3, 5 and 6 are available with the paper.

The Ensembl Gene entry used for the generation of MPC1 conditional knockout with tetO knock-in was ENSMUSG00000023861.

The Ensembl Gene entry used for the generation of MPC2 conditional knockout with tetO knock-in was ENSMUSG00000026568.

Received: 17 February 2020; Accepted: 7 August 2020;

Published online: 26 October 2020

\section{References}

1. Lopaschuk, G. D., Belke, D. D., Gamble, J., Itoi, T. \& Schonekess, B. O. Regulation of fatty acid oxidation in the mammalian heart in health and disease. Biochim. Biophys. Acta 1213, 263-276 (1994).

2. Kerr, P. M., Suleiman, M. S. \& Halestrap, A. P. Reversal of permeability transition during recovery of hearts from ischemia and its enhancement by pyruvate. Am. J. Physiol. 276, H496-H502 (1999).

3. Barger, P. M. \& Kelly, D. P. Fatty acid utilization in the hypertrophied and failing heart: molecular regulatory mechanisms. Am. J. Med. Sci. 318, 36-42 (1999).

4. Razeghi, P. et al. Metabolic gene expression in fetal and failing human heart. Circulation 104, 2923-2931 (2001).

5. Oliver, M. F., Kurien, V. A. \& Greenwood, T. W. Relation between serum-free-fatty-acids and arrhythmias and death after acute myocardial infarction. Lancet 1, 710-715 (1968).
6. Jung, W. I. et al. ${ }^{31} \mathrm{P}$ NMR spectroscopy detects metabolic abnormalities in asymptomatic patients with hypertrophic cardiomyopathy. Circulation 97, 2536-2542 (1998).

7. Nascimben, L. et al. Mechanisms for increased glycolysis in the hypertrophied rat heart. Hypertension 44, 662-667 (2004).

8. Kagaya, Y. et al. Effects of long-term pressure overload on regional myocardial glucose and free fatty acid uptake in rats. A quantitative autoradiographic study. Circulation 81, 1353-1361 (1990).

9. Kolwicz, S. C. Jr. \& Tian, R. Glucose metabolism and cardiac hypertrophy. Cardiovascular Res. 90, 194-201 (2011).

10. Allard, M. F., Schonekess, B. O., Henning, S. L., English, D. R. \& Lopaschuk, G. D. Contribution of oxidative metabolism and glycolysis to ATP production in hypertrophied hearts. Am. J. Physiol. 267, H742-H750 (1994).

11. Sorokina, N. et al. Recruitment of compensatory pathways to sustain oxidative flux with reduced carnitine palmitoyltransferase I activity characterizes inefficiency in energy metabolism in hypertrophied hearts. Circulation 115, 2033-2041 (2007).

12. Taegtmeyer, H. \& Overturf, M. L. Effects of moderate hypertension on cardiac function and metabolism in the rabbit. Hypertension 11, 416-426 (1988)

13. Smith, S. H., Kramer, M. F., Reis, I., Bishop, S. P. \& Ingwall, J. S. Regional changes in creatine kinase and myocyte size in hypertensive and nonhypertensive cardiac hypertrophy. Circulation Res. 67, 1334-1344 (1990).

14. Bricker, D. K. et al. A mitochondrial pyruvate carrier required for pyruvate uptake in yeast, Drosophila, and humans. Science 337, 96-100 (2012).

15. Herzig, S. et al. Identification and functional expression of the mitochondrial pyruvate carrier. Science 337, 93-96 (2012)

16. Schell, J. C. et al. Control of intestinal stem cell function and proliferation by mitochondrial pyruvate metabolism. Nat. Cell Biol. 19, 1027-1036 (2017).

17. Flores, A. et al. Lactate dehydrogenase activity drives hair follicle stem cell activation. Nat. Cell Biol. 19, 1017-1026 (2017).

18. Zhong, Y. et al. Application of mitochondrial pyruvate carrier blocker UK5099 creates metabolic reprogram and greater stem-like properties in LnCap prostate cancer cells in vitro. Oncotarget 6, 37758-37769 (2015).

19. Li, X. et al. Mitochondrial pyruvate carrier function determines cell stemness and metabolic reprogramming in cancer cells. Oncotarget $\mathbf{8}$, 46363-46380 (2017).

20. Schell, J. C. et al. A role for the mitochondrial pyruvate carrier as a repressor of the Warburg effect and colon cancer cell growth. Mol. Cell 56, 400-413 (2014)

21. Li, X. et al. MPC1 and MPC2 expressions are associated with favorable clinical outcomes in prostate cancer. BMC Cancer 16, 894 (2016).

22. Xiao, B. et al. Downregulation of COUP-TFII inhibits glioblastoma growth via targeting MPC1. Oncol. Lett. 15, 9697-9702 (2018).

23. Gray, L. R. et al. Hepatic mitochondrial pyruvate carrier 1 is required for efficient regulation of gluconeogenesis and whole-body glucose homeostasis. Cell Metab. 22, 669-681 (2015).

24. El Alaoui-Talibi, Z., Guendouz, A., Moravec, M. \& Moravec, J. Control of oxidative metabolism in volume-overloaded rat hearts: effect of propionyl-L-carnitine. Am. J. Physiol. 272, H1615-H1624 (1997).

25. Wambolt, R. B. et al. Glucose utilization and glycogen turnover are accelerated in hypertrophied rat hearts during severe low-flow ischemia. J. Mol. Cell. Cardiol. 31, 493-502 (1999).

26. Zimmer, H. G. Regulation of and intervention into the oxidative pentose phosphate pathway and adenine nucleotide metabolism in the heart. Mol. Cell. Biochem. 160-161, 101-109 (1996).

27. Gupte, S. A. et al. Glucose-6-phosphate dehydrogenase-derived NADPH fuels superoxide production in the failing heart. J. Mol. Cell. Cardiol. 41, 340-349 (2006).

28. Hildyard, J. C., Ammala, C., Dukes, I. D., Thomson, S. A. \& Halestrap, A. P. Identification and characterisation of a new class of highly specific and potent inhibitors of the mitochondrial pyruvate carrier. Biochim. Biophys. Acta 1707, 221-230 (2005)

29. Fernandez-Caggiano, M. et al. Analysis of mitochondrial proteins in the surviving myocardium after ischemia identifies mitochondrial pyruvate carrier expression as possible mediator of tissue viability. Mol. Cell. Proteom. 15, 246-255 (2016).

30. van Bilsen, M., van Nieuwenhoven, F. A. \& van der Vusse, G. J. Metabolic remodelling of the failing heart: beneficial or detrimental? Cardiovasc. Res. 81, 420-428 (2009).

31. McCommis, K. S. et al. Nutritional modulation of heart failure in mitochondrial pyruvate carrier-deficient mice. Nat. Metabol. https://doi. org/10.1038/s42255-020-00296-1 (2020).

32. Zhang, Y. et al. Mitochondrial pyruvate carriers are required for myocardial stress adaptation. Nat. Metabol. https://doi.org/10.1038/ s42255-020-00288-1 (2020).

33. UK Government Home Office. Guidance on the Operation of the Animals (Scientific Procedures) Act 1986 (The Stationery Office, 2014). 
34. National Research Council (US) Committee for the Update of the Guide for the Care and Use of Laboratory Animals. Guide for the Care and Use of Laboratory Animals 8th edn (National Academies Press, 2011)

35. Mackay, G. M., Zheng, L., van den Broek, N. J. \& Gottlieb, E. Analysis of cell metabolism using LC-MS and isotope tracers. Methods Enzymol. 561, 171-196 (2015).

36. Carpenter, K. L. H. et al. ${ }^{13} \mathrm{C}$-labelled microdialysis studies of cerebral metabolism in TBI patients. Eur. J. Pharm. Sci. 16, 87-97 (2014).

\section{Acknowledgements}

This work was supported by the British Heart Foundation, the European Research Council (ERC Advanced award) and the Medical Research Council. P.E. is supported by The Barts Charity Cardiovascular Programme Award G00913. We also thank K. Hartmann for her technical assistance and Biobank of 'A Coruña' (XXIAC-Instituto de Investigación Biomédica de A Coruña) for providing healthy heart tissue samples. T.E. acknowledges support from NIHR Biomedical Research Centre at Guy's and St Thomas' NHS Foundation Trust and KCL; the Centre of Excellence in Medical Engineering funded by the Welcome Trust and EPSRC (WT 088641/Z/09/Z) and the KCL

Comprehensive Cancer Imaging Centre funded by the Cancer Research UK (CRUK) and EPSRC in association with MRC and DoH. We acknowledge the metabolic flux analysis facility of the Barts School of Medicine and Dentistry created with the support of the Barts and the London Charity, grant MGU0401.

\section{Author contributions}

P.E. and M.F.-C. conceived the project. M.F.-C., A.K., A.A.F. and O.P. conducted experiments. S.K. performed histology. K.B. and V.M. conducted the C13 flux metabolomics using LC-MS analysis. N.D., M.G.C.-L. and M.G.V. collected human tissue samples and clinical data. M.F.-C. and T.R.E. analysed the metabolomic data. P.E. and M.F.-C. wrote the manuscript. All authors discussed the results and reviewed the manuscript.

\section{Competing interests}

The authors declare no competing interests.

\section{Additional information}

Extended data is available for this paper at https://doi.org/10.1038/s42255-020-00276-5. Supplementary information is available for this paper at https://doi.org/10.1038/ s42255-020-00276-5.

Correspondence and requests for materials should be addressed to M.F.-C. or P.E. Peer review information Primary Handling Editor: George Caputa.

Reprints and permissions information is available at www.nature.com/reprints.

Publisher's note Springer Nature remains neutral with regard to jurisdictional claims in published maps and institutional affiliations.

(c) The Author(s), under exclusive licence to Springer Nature Limited 2020 
A

\section{DAYS Ang II}
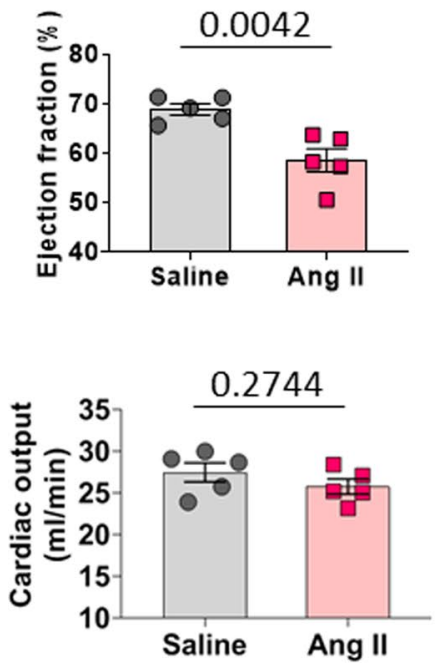

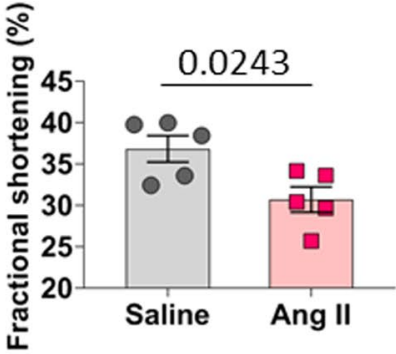

0.0243

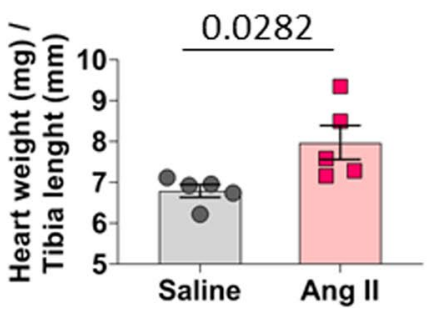

B

\section{DAYS TAC}
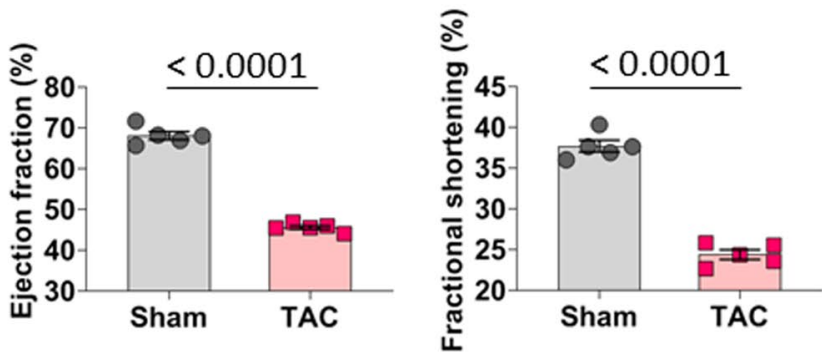

Extended Data Fig. 1 | a, Cardiac function of wild type mice injected with saline or angiotensin presented as ejection fraction, fractional shortening, cardiac output and stroke volume. Development of cardiac hypertrophy was indexed by the heart weight/tibia length ratio $(n=5$ mice/group). b, Cardiac function of wild type mice subjected to a sham or thoracic aortic constriction operation presented as ejection fraction, fractional shortening, cardiac output and stroke volume. Development of cardiac hypertrophy was indexed by the heart weight/tibia length ratio ( $n=5$ mice/group). All statistical significances $(\mathrm{P}<0.05)$ were calculated using unpaired, two-tailed, Student's t-tests. Data are presented as mean \pm S.E.M. 

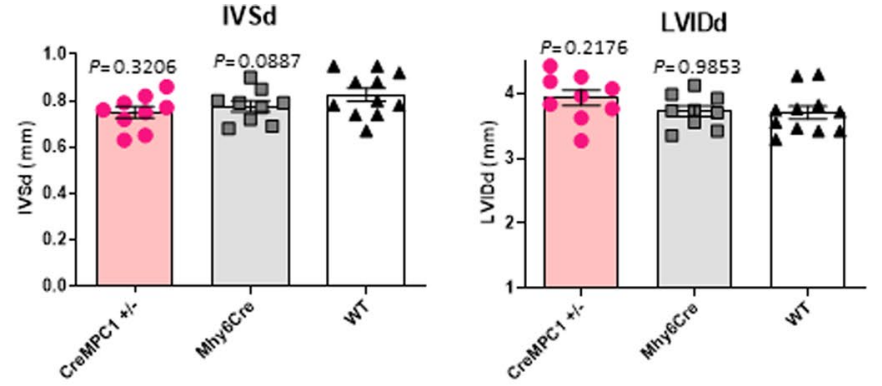

LVPWd

Vol d
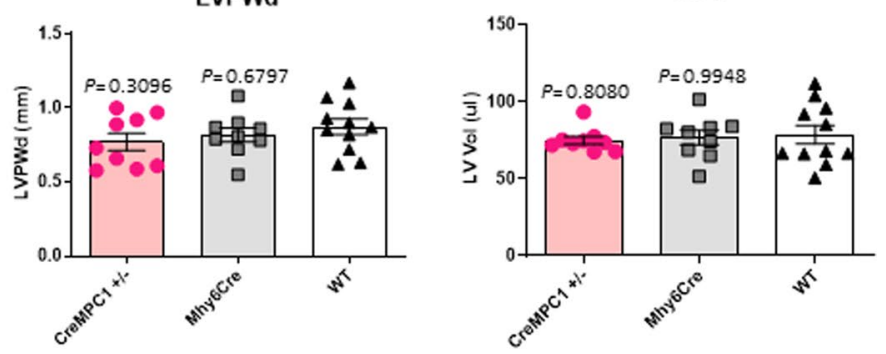

IVSs

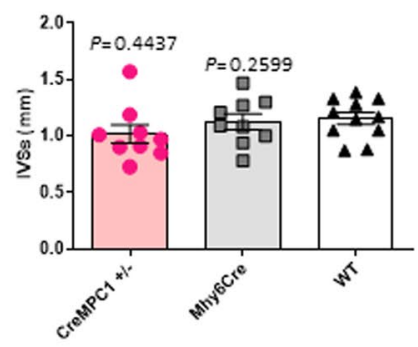

LVIDs

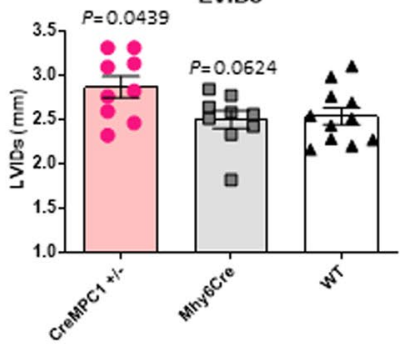

LVPWs

Vol s
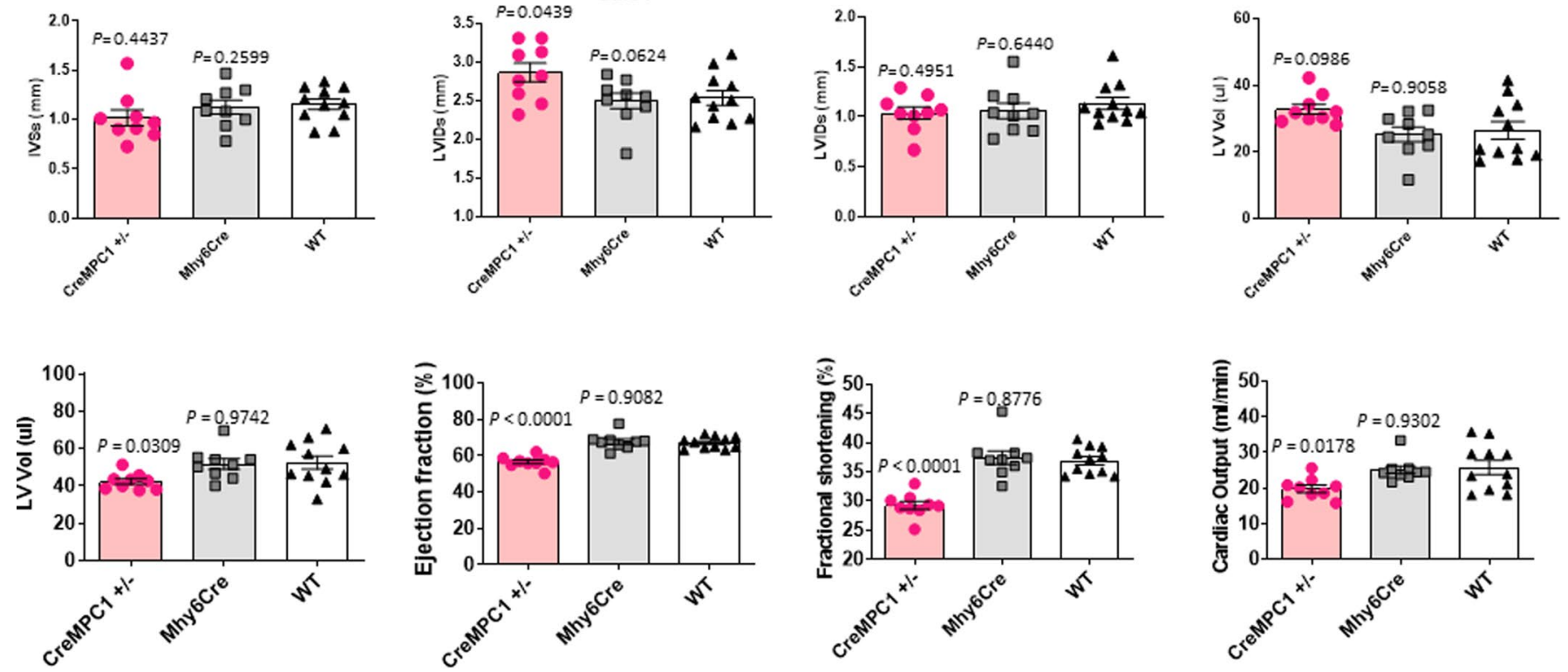

Extended Data Fig. 2 | Cardiac function of partial MPC1 KO (-/ + CreMPC1), Mhy6Cre control and wild type mice was indexed using echocardiography. Cardiac function is presented as interventricular septum thickness at end-diastole and end systole (IVSd and IVSs), left ventricular internal-diastolic (LVIDd), internal-systolic dimension (LVIDs), left ventricular posterior wall in diastole and systole (LVPWd and LVPWs), end diastolic and end systolic volume (Vol d and Vol s), stroke volume, ejection fraction, and fractional shortening ( $n=9-10$ mice/group). Statistical significances $(P<0.05)$ were calculated using one-way ANOVA adjusted using Dunnett's test for multiple comparisons. Data are presented as mean \pm S.E.M. 


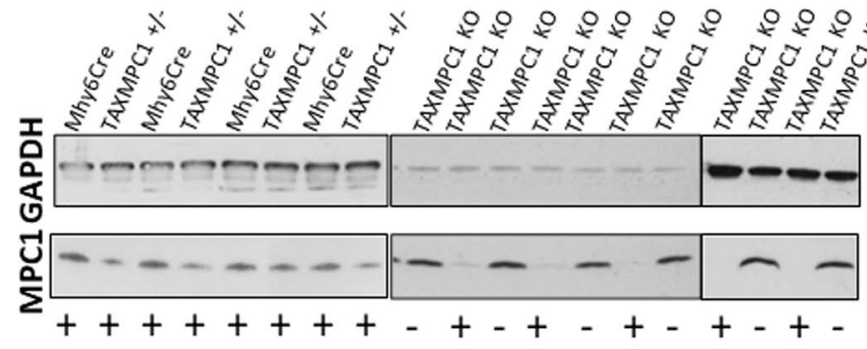

Tamoxifen

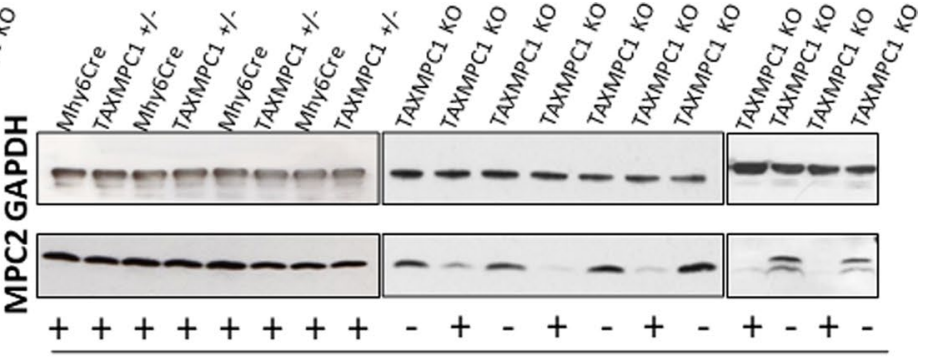

Tamoxifen

\section{MPC1}

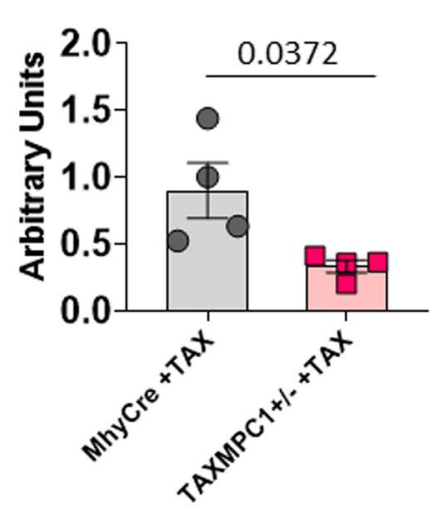

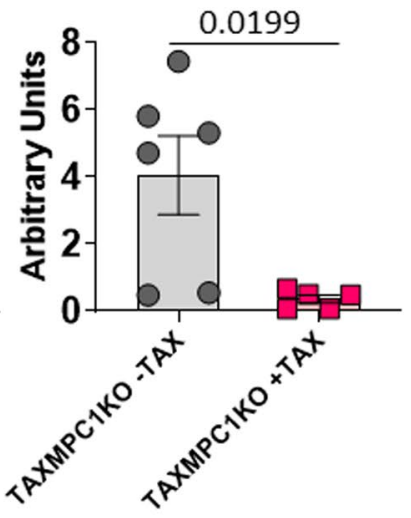
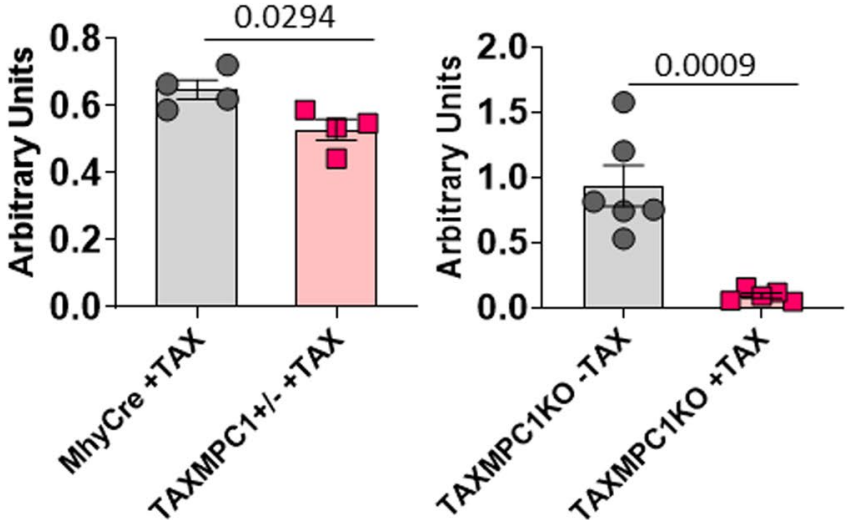

Extended Data Fig. 3 | Western immunoblotting analysis of homozygous (TAXMPC1 KO) or heterozygous (TAXMPC1 ${ }^{+/}$) mouse hearts showed that tamoxifen injection to 8-week-old transgenic TAXMPC1 adults promoted a significant reduction in MPC1 and MPC2 protein abundance $(n=4-6$ biologically independent heart samples/group). All statistical significances $(P<0.05)$ were calculated using unpaired, two-tailed, Student's t-tests. Data are presented as mean \pm S.E.M. 


\section{Control}

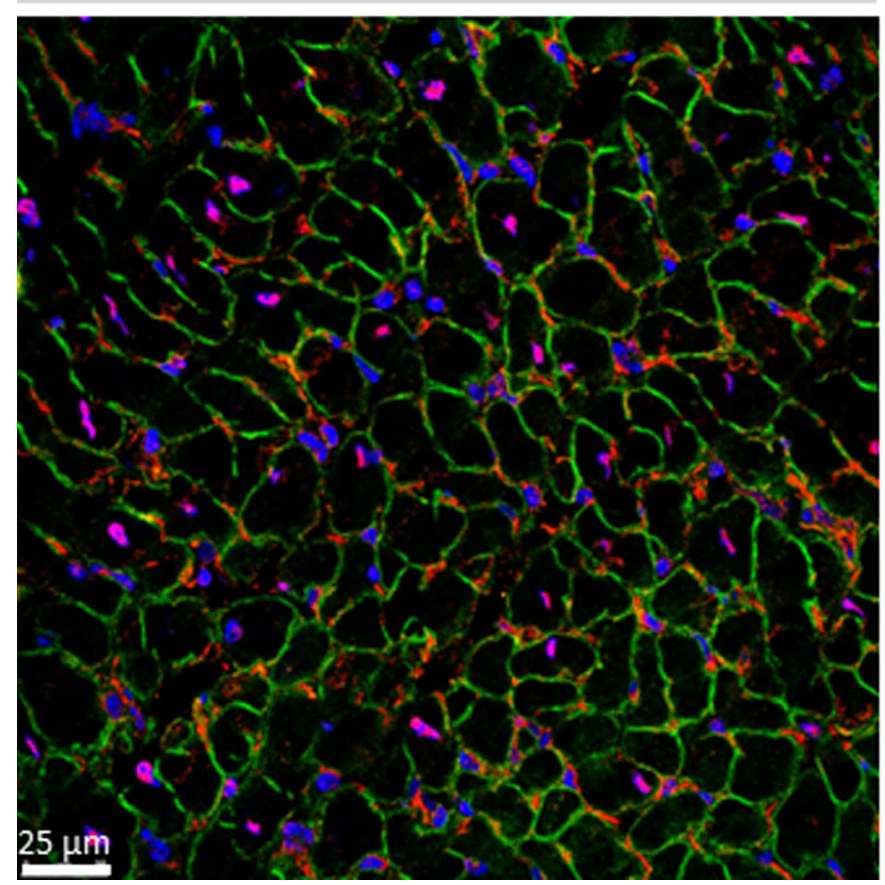

\section{TAXMPC1 +/-}

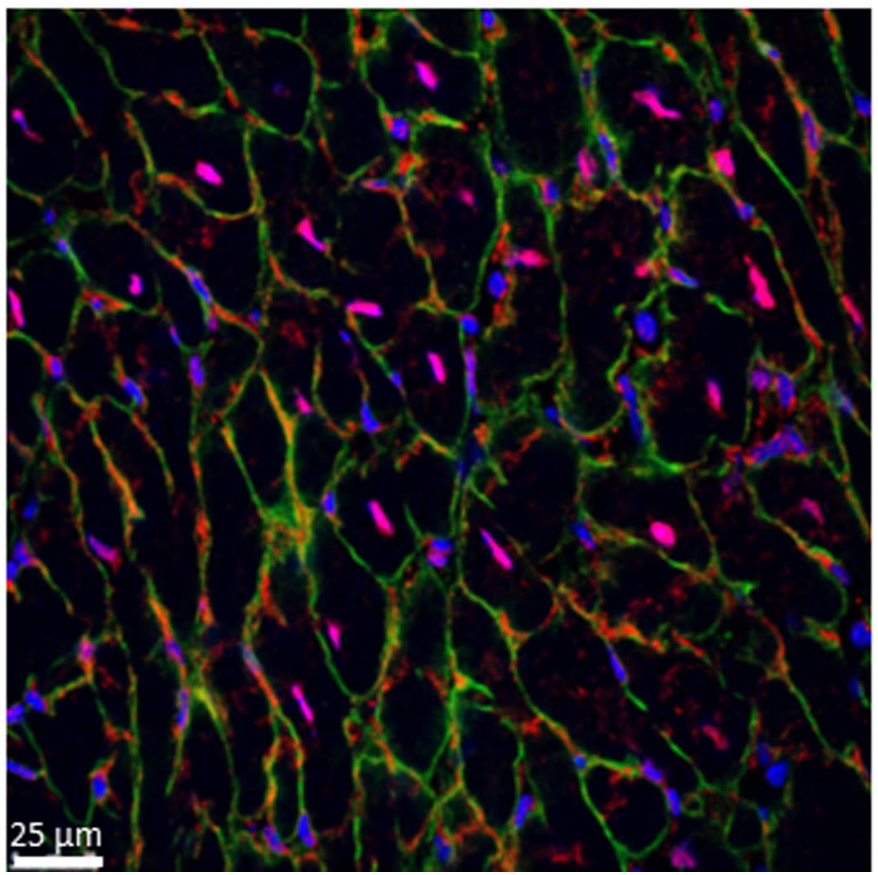

Extended Data Fig. 4 | Immunofluorescence analysis of dystrophin (green), MPC1 (red) and DAPI enabled measurement of cardiomyocyte cross-sectional area. TAXMPC1+/, which are characterised by partial knockout of MPC expression, were hypertrophic as evidenced by the presence of larger cells. (Scale bar represents $25 \mu \mathrm{m}$ ). Representative image for $n=3$ biologically independent hearts/group. 


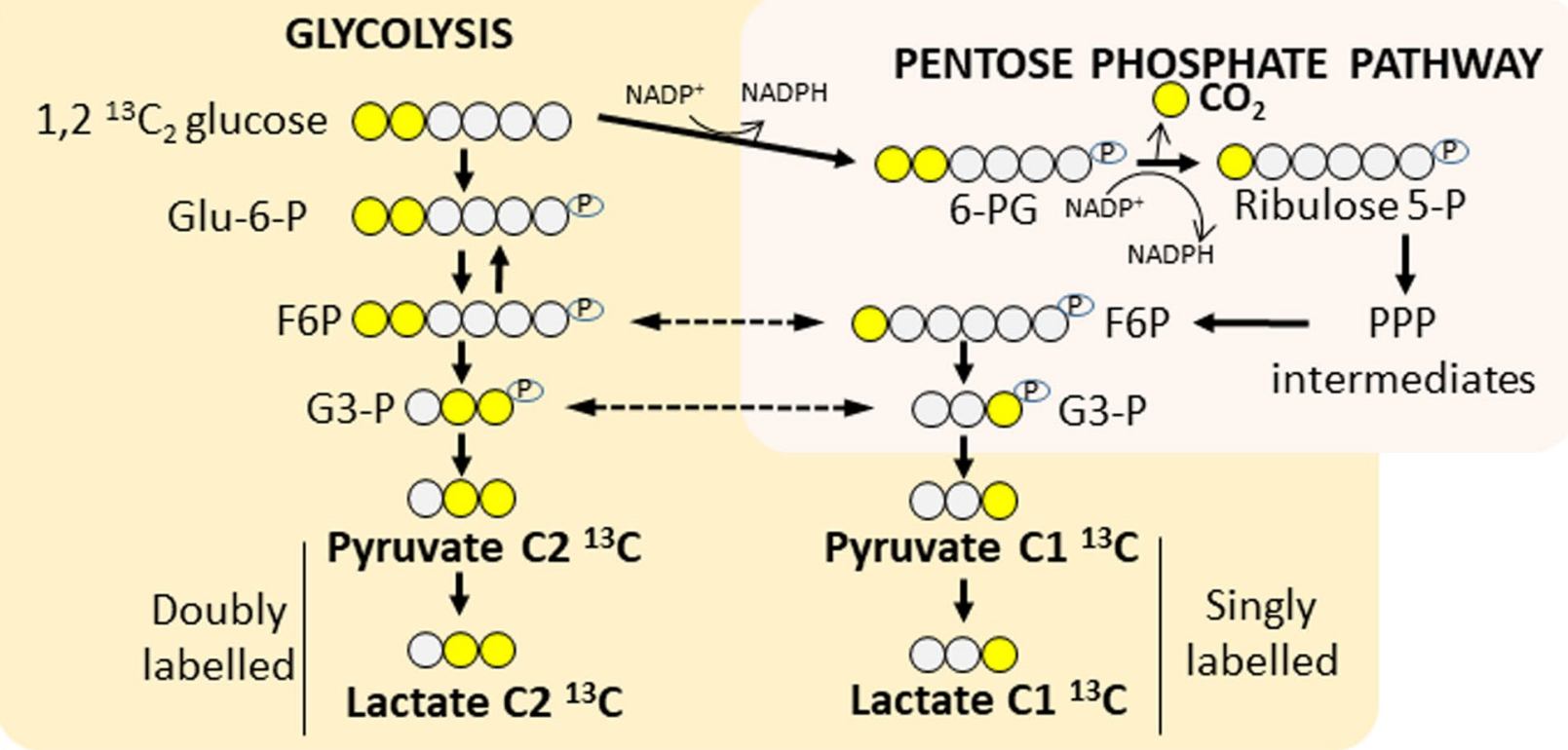

- Control-Cre a TAXMPC1 +/-

Singly labelled Singly labelled

${ }^{13} \mathrm{C}$ pyruvate

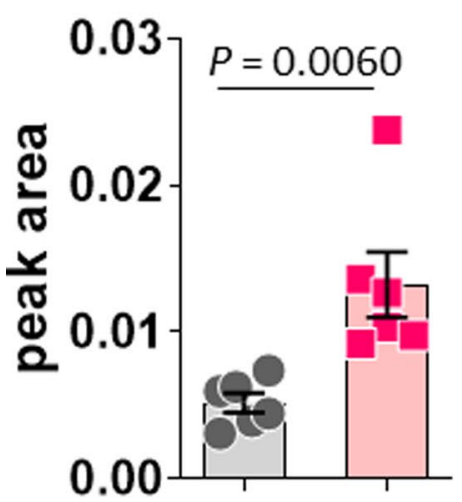

${ }^{13} \mathrm{C}$ lactate

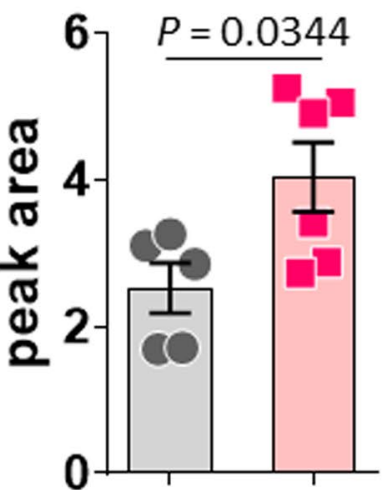

Doubly labelled

${ }^{13} \mathrm{C}$ pyruvate

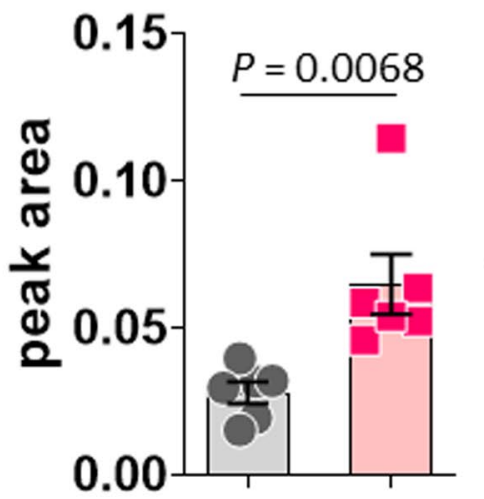

Doubly labelled

${ }^{13} \mathrm{C}$ lactate

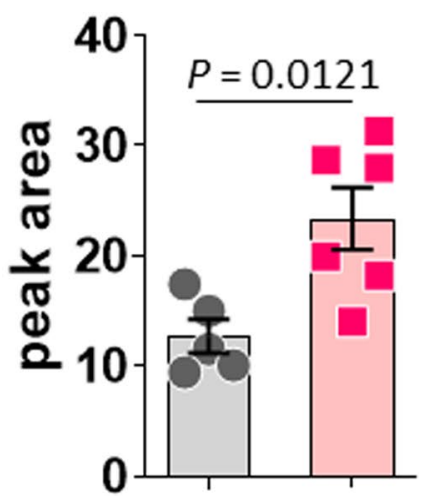

Extended Data Fig. 5 | Diagram adapted from Carpenter et al ${ }^{36}$. to show measurement of flux towards the PPP. TAXMPC1+/- hearts presented an increase in doubly and singly labelled ${ }^{13} \mathrm{C}$ lactate and pyruvate $(n=5-6$ biologically independent heart samples/group). Peak area was normalized by HEPES. All statistical significances $(P<0.05)$ were calculated using unpaired, two-tailed, Student's t-tests. Data are presented as mean \pm S.E.M. 
A

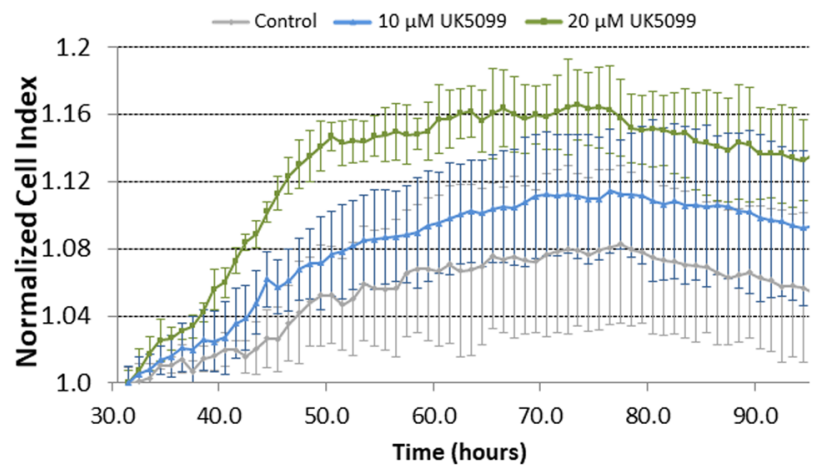

B

NADP $^{+}$

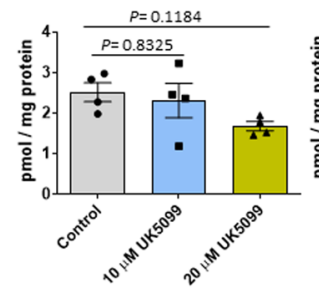

$N A D^{+}$

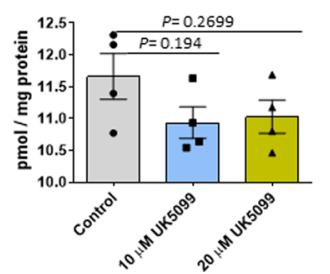

NADPH

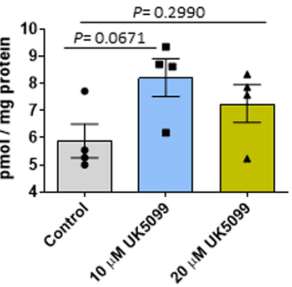

NADH

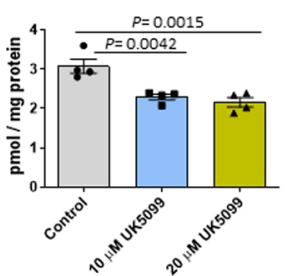

$\mathrm{NADP}^{+}$/ NADPH

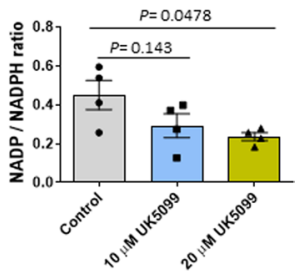

$\mathrm{NAD}^{+} / \mathrm{NADH}$

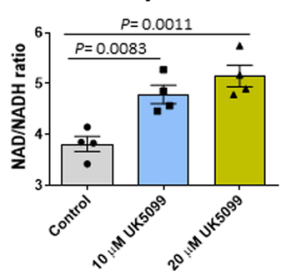

Extended Data Fig. 6 | a, Proliferation and growth of H9C2 cells was increased in response to the mitochondrial pyruvate carrier inhibitor UK5099 (10 $\mu \mathrm{M}$ or $20 \mu \mathrm{M})$. b, H9C2 cells treated with $20 \mu \mathrm{M}$ UK5099 showed a significant reduction in NADP/NADPH and NAD/NADH ratios, whereas only the NAD/ $\mathrm{NADH}$ ratio was significant in $\mathrm{H} 9 \mathrm{C} 2$ cells treated with $10 \mu \mathrm{M}$ UK5099 $(\mathrm{n}=4$ biologically independent cell samples/group). Statistical significances $(\mathrm{P}<0.05)$ were calculated using one-way ANOVA adjusted using Dunnett's test for multiple comparisons. Data are presented as mean \pm S.E.M. 


\section{natureresearch}

Corresponding author(s): Philip Eaton

Last updated by author(s): Aug 6, 2020

\section{Reporting Summary}

Nature Research wishes to improve the reproducibility of the work that we publish. This form provides structure for consistency and transparency in reporting. For further information on Nature Research policies, see Authors \& Referees and the Editorial Policy Checklist.

\section{Statistics}

For all statistical analyses, confirm that the following items are present in the figure legend, table legend, main text, or Methods section.

n/a Confirmed

$\bigotimes$ The exact sample size $(n)$ for each experimental group/condition, given as a discrete number and unit of measurement

$\bigotimes$ A statement on whether measurements were taken from distinct samples or whether the same sample was measured repeatedly

$\triangle$ The statistical test(s) used AND whether they are one- or two-sided

Only common tests should be described solely by name; describe more complex techniques in the Methods section.

Х $\square$ A description of all covariates tested

$\square$ \ A description of any assumptions or corrections, such as tests of normality and adjustment for multiple comparisons

$\triangle$ A full description of the statistical parameters including central tendency (e.g. means) or other basic estimates (e.g. regression coefficient)

AND variation (e.g. standard deviation) or associated estimates of uncertainty (e.g. confidence intervals)

For null hypothesis testing, the test statistic (e.g. $F, t, r$ ) with confidence intervals, effect sizes, degrees of freedom and $P$ value noted

Give $P$ values as exact values whenever suitable.

Х $\square$ For Bayesian analysis, information on the choice of priors and Markov chain Monte Carlo settings

Х $\square$ For hierarchical and complex designs, identification of the appropriate level for tests and full reporting of outcomes

Х $\square$ Estimates of effect sizes (e.g. Cohen's $d$, Pearson's $r$ ), indicating how they were calculated

Our web collection on statistics for biologists contains articles on many of the points above.

\section{Software and code}

Policy information about availability of computer code

Data collection Metabolome measurements were contracted to Human Metabolome Technologies, Inc., Tsuruoka, Japan. Peaks detected by CE-TOFMS and CE-MS/MS were extracted using automatic integration software (MasterHands, Keio University, Tsuruoka, Japan and MassHunter Quantitative Analysis B.04.00, Agilent Technologies, Santa Clara, CA, USA, respectively) to obtain peak information including m/z, migration time (MT), and peak area.

C13 flux metabolomics was performed using a Q Exactive Hybrid Quadrupole-Orbitrap mass spectrometer coupled to a Vanquish UHPLC system (Thermo Fisher Scientific) at QMUL.

Data analysis

Echocardiography was performed with a Vevo 3100 system using a $40 \mathrm{MHz}$ linear probe (Visualsonics, Canada). Ejection fraction, stroke volume, and cardiac output were obtaining from high-resolution motion-mode images at the level of the papillary muscle using the Vevo Software (VisualSonics, software version 1.0.0).

The acquired spectra for C13 flux metabolomics were analyzed using XCalibur Qual Browser and XCalibur Quan Browser software

(Thermo Scientific, software version 4.2).

GraphPad Prism 5.0 and Microsoft Excel (version 16.16.15) was used for statistical test.

Cardiomyocyte area quantification was analyzed using ImageJ (version 1.51W).

Primers were generated using Primer3' (version 0.4.0).

The densitometry of western blots was performed using Gel Pro Analyzer 3.1 (Media Cybernetics USA). 
Policy information about availability of data

All manuscripts must include a data availability statement. This statement should provide the following information, where applicable:

- Accession codes, unique identifiers, or web links for publicly available datasets

- A list of figures that have associated raw data

- A description of any restrictions on data availability

The data that support the findings of this study are available from the corresponding author on request. Human Metabolone Technologies uses the Human Metabome Database (HMDB) (https://hmdb.ca/). The metabolomic data and the HMDB link for each metabolite is available online as Supplementary Table 1 and Supplementary Table 2. Source data for Figs. 1-4 and Supplementary Figs. 1-3 and 5 and 6 are available online.

The Ensembl Gene link used for the generation of MPC1 conditional knockout with tetO knockin is: http://useast.ensembl.org/Mus_musculus/Gene/Summary? $\mathrm{db}=$ core; $\mathrm{g}=$ ENSMUSG00000023861; $\mathrm{r}=17: 8282904-8297661 ; \mathrm{t}=$ ENSMUST00000155364

The Ensembl Gene link used for the generation of MPC2 conditional knockout with tetO knockin is: http://www.ensembl.org/Mus_musculus/Gene/Summary? $\mathrm{db}=$ core;g=ENSMUSG00000026568; $\mathrm{r}=1: 165461037-165481214 ; \mathrm{t}=$ ENSMUST00000027853

\section{Field-specific reporting}

Please select the one below that is the best fit for your research. If you are not sure, read the appropriate sections before making your selection. $\bigotimes$ Life sciences $\quad \square$ Behavioural \& social sciences $\quad \square$ Ecological, evolutionary \& environmental sciences

For a reference copy of the document with all sections, see nature.com/documents/nr-reporting-summary-flat.pdf

\section{Life sciences study design}

All studies must disclose on these points even when the disclosure is negative.

Sample size In all parts of this study we used the minimum number of mice per experiment and human samples that allowed robust and statistically results to be obtained based on previous studies (Kong CHT et al. Cardiac-specific overexpression of caveolin-3 preserves t-tubular ICa during heart failure in mice. Exp Physiol. 2019;104(5):654-666.; Fernández-Caggiano M, et al. Analysis of Mitochondrial Proteins in the surviving myocardium after ischemia identifies mitochondrial pyruvate carrier expression as possible mediator of tissue viability. Mol Cell Proteomics. 2016;15(1):246-255.)

Data exclusions No data were excluded from the analyses.

Replication All analyses were done using at least 3 independent biological replicates/mice and repeated at least twice in separate experiments at different time points. All attempts at replication were successful.

Randomization Animals and samples were randomized and we were not aware of any covariates to control for.

Blinding Data analysis performed blind to the phenotype.

\section{Reporting for specific materials, systems and methods}

We require information from authors about some types of materials, experimental systems and methods used in many studies. Here, indicate whether each material, system or method listed is relevant to your study. If you are not sure if a list item applies to your research, read the appropriate section before selecting a response.

\begin{tabular}{l|l}
\multicolumn{3}{l}{ Materials \& experimental sys } \\
\hline $\mathrm{n} / \mathrm{a}$ & Involved in the study \\
\hline & $\bigotimes$ Antibodies \\
$\square$ & $\bigotimes$ Eukaryotic cell lines \\
$\square$ & $\bigotimes$ Palaeontology \\
$\square$ & $\bigotimes$ Animals and other organisms \\
$\searrow$ & $\square$ Clinical data
\end{tabular}

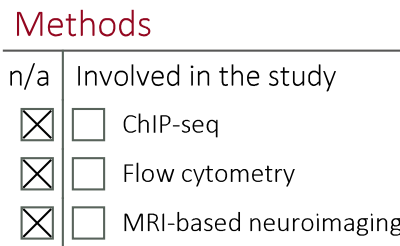

\section{Antibodies}

Antibodies used 
Horseradish peroxidase-coupled anti-rabbit (\#7074, Cell Signalling) IgG secondary antibody https://www.cellsignal.co.uk/ products/secondary-antibodies/anti-rabbit-igg-hrp-linked-antibody/7074

Anti-dystrophin antibody (MAB1645, EMD Millipore) https://www.merckmillipore.com/GB/en/product/Anti-DystrophinAntibody-clone-1808,MM_NF-MAB1645?ReferrerURL=https\%3A\%2F\%2Fwww.google.com\%2F\&bd=1

Antibodies were bought from reputable sources and performed in a manner consistent with the protein they should bind to.

MPC1 (Cell Signalling, \#14462)(6 citations)

https://www.citeab.com/antibodies/2444857-14462-mpc1-d2I9i-rabbit-mab?utm campaign=Widget+All

+Citations\&utm_medium=Widget\&utm_source=Cell+Signaling+Technology\&utm_term=Cell+Signaling+Technology

MPC2 (Cell Signalling, \#46141) (7citations)

https://www.citeab.com/antibodies/3368835-46141-mpc2-d4i7g-rabbit-mab?utm_campaign=Widget+All

+Citations\&utm_medium=Widget\&utm_source=Cell+Signaling+Technology\&utm_term=Cell+Signaling+Technology

GAPDH (Cell Signalling, D16H11)(95 citations)

https://www.citeab.com/antibodies/125652-8884-gapdh-d16h11-xp-rabbit-mab-hrp-conjugate?utm_campaign=Widget+All

+Citations\&utm_medium=Widget\&utm_source=Cell+Signaling+Technology\&utm_term=Cell+Signaling+Technology

\section{Eukaryotic cell lines}

Policy information about cell lines

Cell line source(s)

H9C2 (2-1) Cell Line from rat (BDIX heart myoblast, 88092904) Sigma Aldrich

Authentication

None as directly purchased from Sigma and visual inspection and behavior was consistent with expectations for these cells.

Mycoplasma contamination

Cells tested negative for mycoplasma.

Commonly misidentified lines

(See ICLAC register)

This cell line is not listed in the ICLAC database.

\section{Animals and other organisms}

Policy information about studies involving animals; ARRIVE guidelines recommended for reporting animal research

Laboratory animals

Male mice between 10-13 weeks of age were used for experiments. Animals were kept under pathogen-free conditions, $12 \mathrm{~h}$ light-dark cycle, controlled humidity $(\sim 40 \%)$, temperature $\left(20-22^{\circ} \mathrm{C}\right)$, and fed chow and water ad libitum. Transgenic MPC1 and MPC2 FAST (Flexible Accelerated STOP Tetracycline Operator (tetO)-knock-in) mouse lines were generated by Ingenious Targeting Laboratory (iTL) technologies (Ronkonkoma, New York).

Cardiac-specific mouse transgenic lines FVB-Tg(Myh6-cre)2182Mds/J (Jax Labs, stock: 011037) and FVB.Cg-Tg(Myh6-tTA)6Smbf/J (Jax Labs, stock: 003170) were backcrossed for $>10$ generations into the C57BI/6 background (Jax Labs, Stock: 005304). These mice were then crossed with MPC1 or MPC2 FAST transgenic mice to generate cardiac specific MPC KO (Mhy6CreMPC1 and Mhy6CreMPC2) or overexpressers (Mhy6tTAMPC1 or Mhy6tTAMPC2). The tamoxifen-inducible MPC1 KO were generated by crossing MPC1 FAST transgenic mice with B6.FVB(129)-A1cfTg(Myh6-cre/Esr1*)1Jmk/J (Jax Labs, stock: 005657).

Wild animals

The study did not involve wild animals.

Field-collected samples

The study did not involve samples collected from the field.

Ethics oversight

This investigation was performed in accordance with the Home Office Guidance on the Operation of the Animals (Scientific Procedures) Act 1986, published by Her Majesty's Stationery Office (London, UK). Animals were maintained humanely in compliance with the "Principles of Laboratory Animal Care" formulated by the National Society for Medical Research and the Guide for Care and Use of Laboratory Animals prepared by the National Academy of Sciences and published by the National Institutes of Health (NIH Pub. No. 85-23, revised 1985). All animal protocols were approved both by the local King's College Ethical Review Process Committee and by the UK Government Home Office (Animals Scientific Procedures Group).

Note that full information on the approval of the study protocol must also be provided in the manuscript.

\section{Human research participants}

\section{Policy information about studies involving human research participants}

Population characteristics

Left ventricle biopsies were obtained from the explanted hearts of patients with hypertrophic cardiomyopathy undergoing cardiac transplantation at the A Coruña Hospital. The six individuals with healthy myocardium ( 2 males and 4 females) had a median age of 52 years. The six patients with hypertrophic cardiomyopathy ( 3 males and 3 females) had a median age of 52 .

Recruitment

Hypertrophic cardiomyopathy patients were identified following the American College of Cardiology and American Heart Association clinical guidelines. Left ventricle biopsies of healthy hearts were obtained from unused donor hearts from the $A$ Coruña Hospital following guidelines of Spanish Royal Decrees 2070/1999 and 1301/2006, which regulates the acquisition and 
use of human tissues for clinical and research purposes. We are unaware of any self-selection or other biases that might impact the results.

Ethics oversight

The study was conducted according to Spanish Law for Biomedical Research (Law 14/2007-3 of July) and was compliant with the Declaration of Helsinki. The study and the use of archive samples for this project were approved by the Research Ethics Committee of Galicia. Written, informed consent was obtained from all the individuals involved in this study.

Note that full information on the approval of the study protocol must also be provided in the manuscript. 